\title{
Ka-Band Dual Copolarized Empirical Model for the Sea Surface Radar Cross Section
}

\author{
Yurovsky Yury Yu ${ }^{1}$, Kudryavtsev Vladimir N. ${ }^{1,2}$, Grodsky Semyon A. ${ }^{3}$, Chapron Bertrand 4
}

${ }^{1}$ RAS, Fed State Budget Sci Inst, Inst Marine Hydrophys, Sevastopol 299011, Ukraine.

2 Russian State Hydrometeorol Univ, Satellite Oceanog Lab, St Petersburg 195196, Russia.

3 Univ Maryland, Dept Atmospher \& Ocean Sci, College Pk, MD 20742 USA.

${ }^{4}$ Inst Francais Rech Exploitat Mer, F-29280 Plouzane, France.

Email addresses : yyyurovsky@gmail.com; kudr@rshu.ru ; senya@umd.edu ; bertrand.chapron@ifremer.fr

\begin{abstract}
:
This paper presents dual copolarized (PP) (VV and $\mathrm{HH}$ ) Ka-band sea surface backscattering measurements taken from the Black Sea research platform at incidence angles ranging from 25 degrees to 65 degrees and in the wind speed range from 3 to $18 \mathrm{~m} / \mathrm{s}$. These measurements are corrected for radar antenna pattern and geometry of observations. The resulting normalized radar cross section (NRCS) is parameterized in a form of truncated azimuthal Fourier series with coefficients dependent on the incidence angle and wind speed. This dual PP empirical model (KaDPMod) is consistent with the Ku-band NSCAT4 model. However, some remarkable differences are revealed. They are apparent when analyzed using a decomposition of $\mathrm{VV}$ and $\mathrm{HH}$ measurements into polarized Bragg backscattering (polarization difference, $\mathrm{PD}=\mathrm{VV}-\mathrm{HH}$ ) and nonpolarized (NP) backscattering from breaking waves. The polarization difference (PD) has strong azimuth and wind dependencies, with the wind exponent ranging from 2.5 to 3. The saturation wave spectra derived from multifrequency PD (based on KaDPMod and Ku- and C-band empirical models) have a notable peak in the capillary-gravity range. The relative contribution of NP radar return to the Ka-band NRCS is significant. In the upwind direction, it reaches up to $60 \%-80 \%$ and $25 \%$ $50 \%$ for $\mathrm{HH}$ and $\mathrm{VV}$, respectively. It is found that the NP wind exponent is lower than that for Bragg backscattering. Therefore, the relative contribution of the NP to Ka-band NRCS decreases with increasing wind speed at both polarizations. Such a behavior is the opposite of that observed in the Ku-band.
\end{abstract}

Keywords : Capillary waves, cross section, radar backscattering, sea surface, wave breaking 
Active microwave sensors are powerful tools for the ocean remote sensing. Most of them operate in decimeter or centimeter radio bands (L-, C-, X-, Ku-band) and have typical ground footprints of the order of tens kilometers

Manuscript received. The work has been supported by the Russian Science Foundation under grant 15-17-20020. The work of S. Grodsky was supported by NASA/Physical Oceanography grant NNX15AG40G.

Yu.Yu. Yurovsky and V.N. Kudryavtsev are with the Federal State Budget Scientific Institution "Marine Hydrophysical Institute of RAS", Kapitanskaya 2, Sevastopol, Russia, 299011 (e-mail: yyyurovsky@gmail.com).

V.N. Kudryavtsev is also with the Satellite Oceanography Laboratory, Russian State Hydrometeorological University, Malookhtinsky prospect 98, Saint Petersburg, Russia (e-mail: kudr@rshu.ru).

S.A. Grodsky is with the University of Maryland, Department of Atmospheric and Oceanic Science, College Park, USA (e-mail: senya@umd.edu).

B. Chapron is with the Institut Français de Recherche pour l'Exploitation de la Mer, 29280, Plouzané, France (e-mail: Bertrand.Chapron@ifremer.fr). 
(for real aperture sensors). A demand for higher spatial resolution and detection of coastal phenomena requires exploration of higher radar frequencies, which is also expected to improve surface topography accuracy. The first spaceborne Ka-band (35.75 GHz) altimeter, AltiKa [1], launched in 2013, has demostrated almost a factor of three improvement in sea level retrieval accuracy in comparison with the previous Ku-band TOPEX/Poseidon. The launch of a new Ka-band interferometer, KaRIN, designed for the Surface Water Ocean Topography mission, scheduled for 2019, is expected to provide the first radar mesoscale ocean survey [2]. Atmospheric attenuation, which becomes increasingly important in millimeter radio bands, needs to be accounted for in ocean applications. On the other hand, measurements in this band provide information on the atmospheric state, and are used for rainfall retrieval by the Global Precipitation Measurement mission dual frequency Ku/Ka-band radar [3].

It is expected that future ocean applications of millimeter range radars will combine their higher spatial resolution with Synthetic Aperture Radar (SAR) capabilities [4]-[6]. In fact Ka-band airborne SAR instruments have already demonstrated incredible spatial resolution of down to $10 \mathrm{~cm}$, significantly exceeding the resolution of traditional C- and X-band instruments [7].

Besides various applications based on detection of backscattering intensity, radar Doppler frequency spectra can provide direct measurements of the line-of-sight projection of surface currents over the global ocean [8], [9]. The conical rotating Doppler scatterometer configuration enables instruments of this type to retrieve surface current velocity, while the use of the higher Ka-band frequency should increase the spatial resolution and retrieval accuracy by a factor of three [10].

Development and understanding of Ka-band capabilities require accurate characterization of Geophysical Model Functions (GMFs) relating the sea surface cross-section and the Doppler frequency shift as a function of environmental variables and the observation geometry. To date, the most well established GMFs are developed for the lower radar frequencies, e.g. C-band CMOD5.N [11], Ku-band QSCAT-1 [12], and NSCAT [13], [14].

Ka-band measurements for various observation geometries are still quite rare. A complete tabulated model for VV and HH polarizations has been proposed in [15] (referred hereinafter as MOSN'86). That table was based on airborne measurements in a wide range of incidence angles (from $0^{\circ}$ to $70^{\circ}$ ) and wind speeds (from $3.2 \mathrm{~m} / \mathrm{s}$ to 17.2 $\mathrm{m} / \mathrm{s}$ ). A corresponding MOSN'86 analytical parametrization [16] is available only for VV polarization and only for fixed incidence angles of $30^{\circ}, 40^{\circ}, 50^{\circ}$, and $60^{\circ}$.

However, a number of independent measurements show significant deviations from the MOSN'86 (Fig. 1). In particular, a notable positive deviation of about 5-6 dB [17]-[19] at near-nadir angles has been observed which has been attributed to uncertainties in the absolute calibration. The absolute calibration was made in [15] separately for transmitter and receiver, but not for the entire system. At moderate incidence angles, both field data [20] and wind-wave tank data [21], [22] show backscattering levels exceeding those described in MOSN'86.

Ka-band backscattering data are thus not numerous and those that do exist demonstrate significant scatter. This paper aims to narrow this scatter using Ka-band Doppler dual co-polarized (VV and $\mathrm{HH}$ ) data collected during a number of field experiments carried out from the Black Sea research platform during 2009-2015. Observed features of Ka-band backscattering are discussed and compared with independent Ka- and Ku-band radar measurements. 


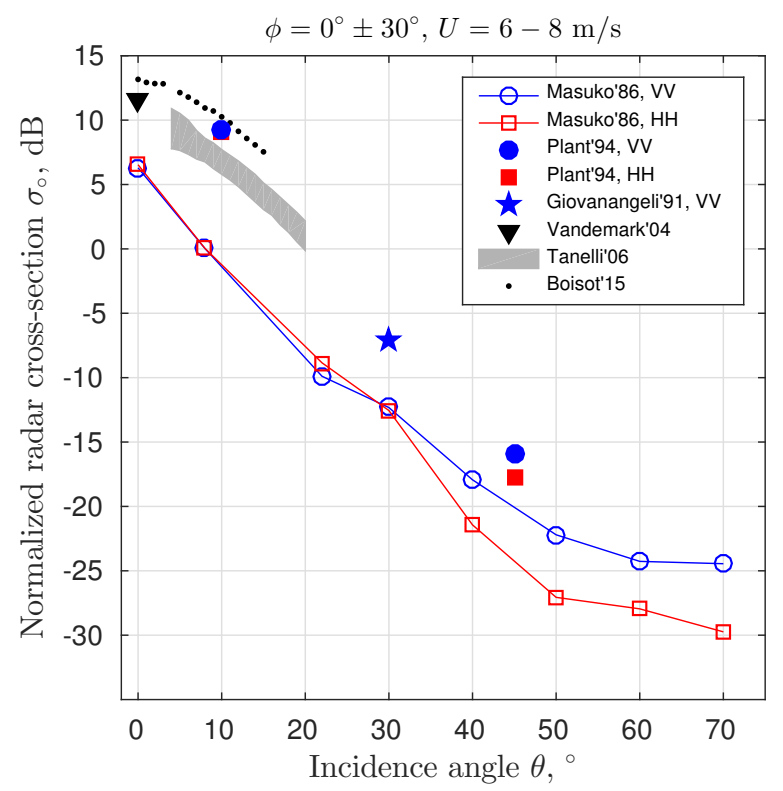

Fig. 1. Ka-band upwind NRCS versus incidence angle at $6-8 \mathrm{~m} / \mathrm{s}$ wind speed. Blue is VV polarization, red is HH polarization, black is unknown (no matter) polarization. Data sources are listed by the first author and publication year.

\section{EXPERIMENT}

\section{A. Equipment}

The measurements are taken from a Research platform operated by the Marine Hydrophysical Institute, which is located in the Black Sea 600 offshore of Katsively ( $44^{\circ} 23^{\prime} 35^{\prime \prime}$ N, $33^{\circ} 59^{\prime} 04^{\prime \prime}$ E) in a $25 \mathrm{~m}$ to $30 \mathrm{~m}$ deep water (Fig. 2).

The experiments were carried out using Ka-band (37.5 GHz, $8 \mathrm{~mm}$ wavelength) continuous wave Doppler radar. The radar has two conical horn antennae for transmitting and receiving. Antenna horn axes are parallel and separated by $35 \mathrm{~cm}$. The length of the horn axis is $38 \mathrm{~cm}$, and the diameter of the aperture is $16.5 \mathrm{~cm}$. The polarization plane of the Gunn diode-based transmitter is rotated by $45^{\circ}$ from the radar incidence plane so that vertical and horizontal components are radiated simultaneously. The receiver has two separate vertical and horizontal channels. Such a hybrid radar design does not allow discrimination between co-polarized and cross-polarized (CP) signals. In fact, vertical and horizontal channels receive a mixture of $\mathrm{VV}+\mathrm{HV}$ and $\mathrm{HH}+\mathrm{VH}$ components, respectively. However at wind speeds below $25 \mathrm{~m} / \mathrm{s}$, the CP signal is much lower than the co-polarized (VV and HH) signals [23]-[25]. Hence, we ignore the $\mathrm{CP}$ contribution and consider the hybrid vertical and horizontal signals as "pure" co-polarized, VV and HH, signals (see Appendix B for more discussion).

The radar has a built-in high-pass filter with a $5 \mathrm{~Hz}$ frequency cut-off to avoid reflections from static objects. Inphase and quadrature channels for each polarization are digitized at $40 \mathrm{kHz}$ using a PC-based 14-bit analog-to-digital converter. 


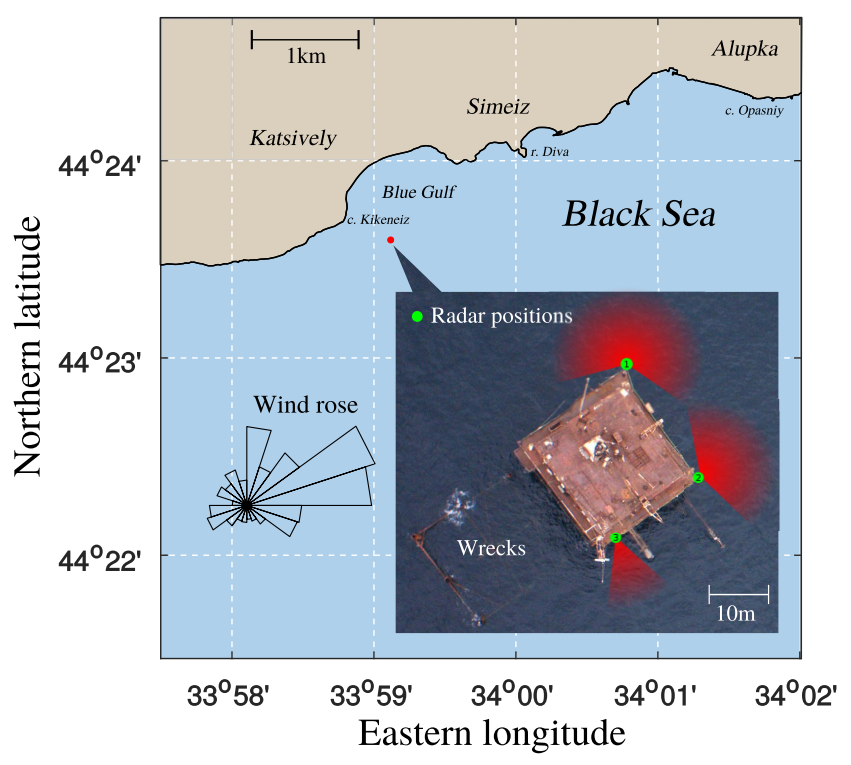

Fig. 2. The Black Sea Research platform site and top view. Radar positions are marked by green circles, possible azimuths at each position are shown by red sectors. Wind rose is plotted for the boreal autumn. "Wrecks" indicate location of the platform module destroyed by storms.

Wind speed and direction are measured using a standard cup anemometer and wind vane installed on top of the platform mast at $21 \mathrm{~m}$ above the sea surface. Air temperature, pressure, and humidity at $21 \mathrm{~m}$ height and water temperature at $3 \mathrm{~m}$ depth are continuously monitored by commercial weather station (Davis Vantage Pro2 6152). Supplementary wave information is obtained using a wire wave gauge installed from $11 \mathrm{~m}$ long horizontal boom. Neutral wind speed at $10 \mathrm{~m}, U$, is computed from the meteorological observations and near surface water temperature using the COARE3.0 algorithm [26].

\section{B. NRCS Measurement}

A few metal targets (trihedral corner reflectors and spheres of different sizes) were used to calibrate the radar and estimate its two-way patterns (see Appendix A). The radar beam width is not symmetrical ans is wider in the polarization plane $\left(15^{\circ}\right.$ at $-3 \mathrm{~dB}$, Fig. 18 in Appendix A). The calibration was repeatedly performed during each field campaign, but no significant changes were detected.

The backscattered power, $p^{\prime}$, from a stand-alone point-like reference target is determined by the radar equation

$$
p^{\prime}=C \frac{\Gamma^{\prime}}{R^{\prime 4}} \sigma^{\prime}
$$

where $C$ is the calibration constant determined by radar architecture (see Appendix A), $\Gamma^{\prime}$ is the two-way radar pattern in the direction of the target, $R^{\prime}$ is the distance to the target, $\sigma^{\prime}$ is the radar cross-section (RCS) of the target. 
Because the sea surface is a distributed target, its radar backscattering is integrated over the antenna pattern:

$$
p=\frac{C}{R_{0}^{4}} \int \Gamma_{\text {eff }}(x, y) \sigma_{\circ} \mathrm{d} x \mathrm{~d} y=\frac{C}{R_{0}^{4}} \sigma_{\text {eff }},
$$

where $\sigma_{\circ}(x, y)$ is the sea surface normalized RCS (NRCS) that varies spatially depending on the sea surface $\{x, y\}$ coordinates, $\Gamma(x, y)$ is the two-way radar angular pattern in the direction of $\{x, y\}$-point on the surface, $R(x, y)$ is the slant range to $\{x, y\}$-point, $R_{0}$ is the slant range along the radar principal axis, $\Gamma_{\text {eff }}(x, y)=\Gamma(x, y)\left(R_{0} / R(x, y)\right)^{4}$ is the effective pattern accounting for changes in the slant range, and $\sigma_{\text {eff }}$ is the effective RCS of the spatially distributed sea surface target.

For an infinitely narrow radar angular pattern, $\sigma_{\circ}$ is almost constant within the radar surface footprint and can be taken out the integral in (2). However, in our case the radar angular pattern is noticeably wide, and thus the parameter we measure is a convolution of $\sigma_{\circ}(x, y)$ and $\Gamma(x, y)$. Because NRCS dependence on the incidence angle is much stronger than dependence on the azimuth angle, the antenna pattern impact is stronger for $\mathrm{VV}$ than for HH polarization.

The effective (measured) NRCS of the distributed sea surface target is defined as the ratio of effective RCS, $\sigma_{\text {eff }}$, and effective radar footprint, $S_{\text {eff }}=\int \Gamma_{\text {eff }}(x, y) \mathrm{d} x \mathrm{~d} y$, and reads:

$$
\sigma_{\text {off }}=\frac{\sigma_{\mathrm{eff}}}{S_{\mathrm{eff}}}=p \frac{R_{0}^{4}}{C}\left[\int \Gamma(x, y)\left(\frac{R_{0}}{R(x, y)}\right)^{4} \mathrm{~d} x \mathrm{~d} y\right]^{-1} .
$$

Radar internal noise prevents measurements at calm winds and/or high incidence angles. Its level (estimated from clear sky radar backscattering) is subtracted from the measured radar backscattering. Radar data are disregarded if their signal-to-noise ratio is below 1. The noise level of NRCS is estimated from (3) by substituting the received power by its noise variance (Fig. 3). Discontinuity of the NRCS noise curves in Fig. 3 at $\theta=45^{\circ}$ is related to different altitudes used for radar measurement at $\theta<45^{\circ}$ and $\theta>45^{\circ}$. The noise level determines the critical incidence angles and winds for which radar backscattering from the sea surface is detectable. In our observations, the signal-to-noise ratio falls below one at $\theta>70^{\circ}$ for $\mathrm{HH}$ polarization at winds below $5-6 \mathrm{~m} / \mathrm{s}$. Thus the upper limit of $\theta$ for our data is set to $70^{\circ}$.

\section{Data Set}

The radar look direction and the start of the acquisition cycle were chosen manually depending on wind and wave conditions. Typical records last from from $5 \mathrm{~min}$ to $60 \mathrm{~min}$. For the processing purposes, each record was split into 5 min fragments, which we refer to as data samples. The total number of data samples is about 1500 .

Depending on the incidence angle, $\theta$, the radar is installed on top or bottom deck of the platform. The top deck installation (13.5 $\mathrm{m}$ height) is used for incidence angles $0^{\circ} \leq \theta \leq 45^{\circ}$, while the bottom installation (6.5 $\mathrm{m}$ height) is used for higher incidence angles, $\theta>45^{\circ}$. In both cases the far field approximation is applicable.

Assuming that the NRCS is symmetrical relative to the wind direction, the data are folded in the azimuth range, $0^{\circ} \leq \phi \leq 180^{\circ}$. To minimize the impact of platform-induced wind distortions on downwind measurements all 


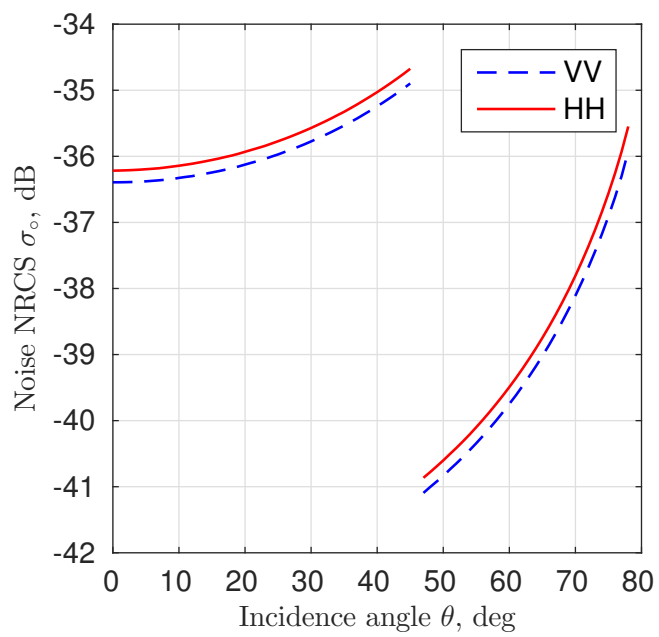

Fig. 3. Noise level of the measured NRCS as a function of the incidence angle. The discontinuity at $\theta=45^{\circ}$ is caused by the difference in radar height at different $\theta$.
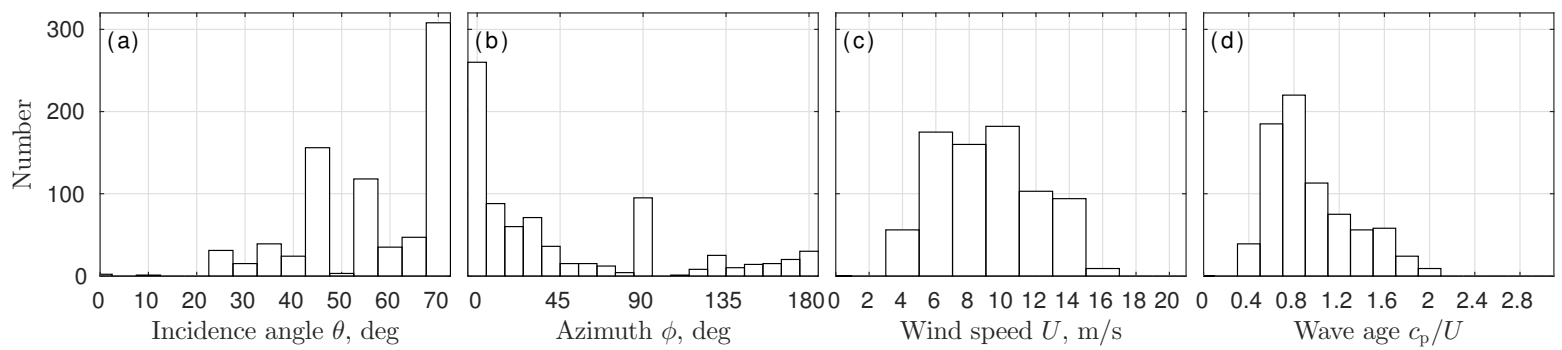

Fig. 4. Data set statistics. Distribution of measurements on (a) incidence angle, (b) radar versus wind azimuth (zero is upwind), (c) wind speed, and (d) wave age.

downwind observations were carried out from the most outlying platform corner, so that the radar beam was never directed towards the platform "shadow" zone (Fig. 2).

Most of the data samples correspond to moderate $\left(45^{\circ}<\theta<55^{\circ}\right)$ and high $\left(\theta=70^{\circ}\right)$ incidence angles, while the near nadir data $\left(\theta<30^{\circ}\right)$ are sparse (Fig. 4a).

Observed wind speed, $U$, varies from $3 \mathrm{~m} / \mathrm{s}$ to $18 \mathrm{~m} / \mathrm{s}$ with the most common speeds falling in the range from 5 $\mathrm{m} / \mathrm{s}$ to $11 \mathrm{~m} / \mathrm{s}$ (Fig. $4 \mathrm{c}$ ). There are three typical wind directions at the platform location: easterly, southwesterly, and northerly (Fig. 2). The latter is removed from the analysis because it corresponds to offshore winds and short ( $\sim 1$ $\mathrm{km}$ ) wave fetches. For easterly or southwesterly winds, the wave fetch is determined by the size of atmospheric synoptic systems and can reach a few hundred kilometers. Our analysis avoids transient wind/wave conditions and focuses on temporally stable conditions typical of the open ocean. In addition, we also exclude cases of the swelldominated sea. Swell-induced strong radar backscattering modulations, which may significantly affect the mean radar signal, are out of the scope of this study. 

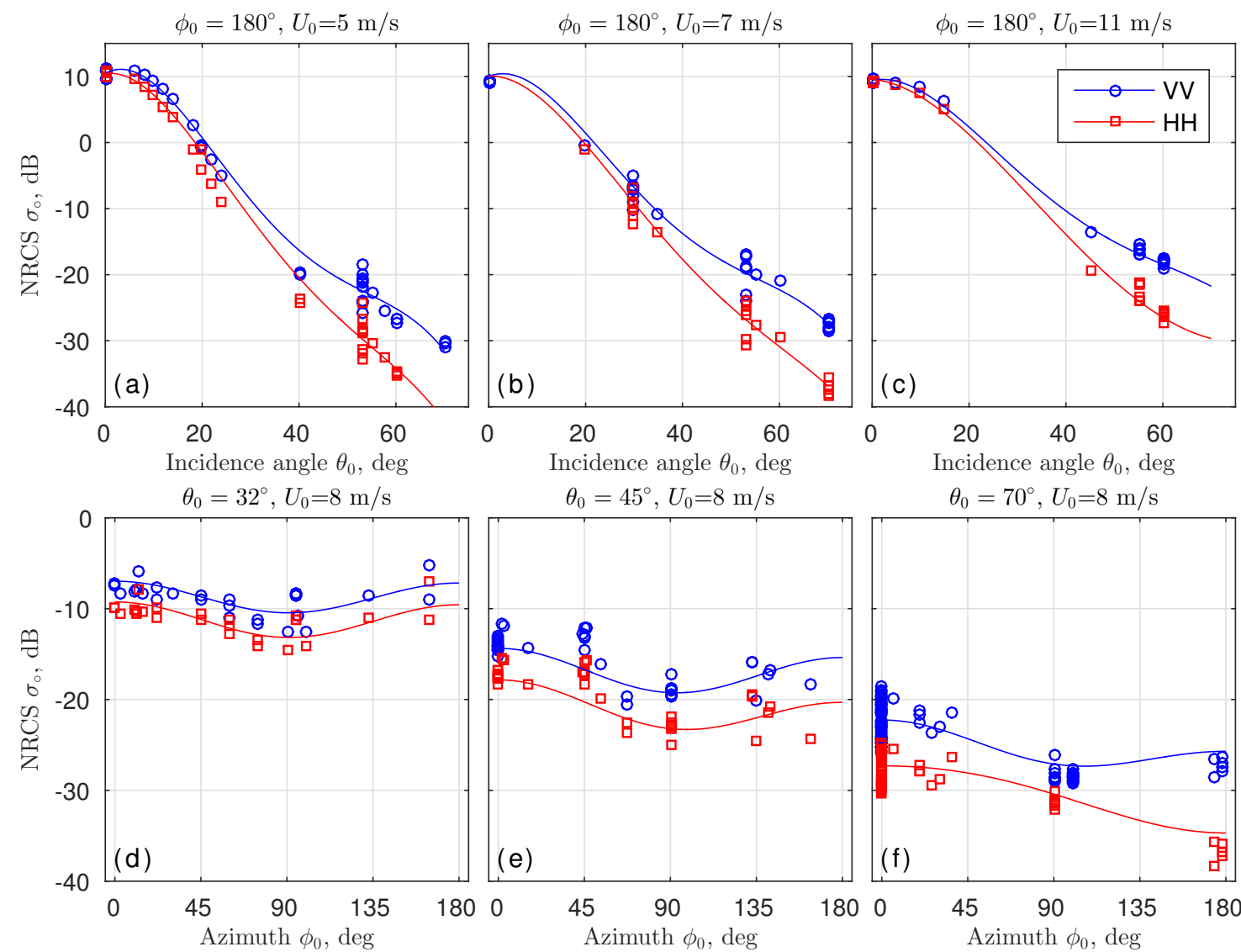

$\theta_{0}=45^{\circ}, U_{0}=8 \mathrm{~m} / \mathrm{s}$

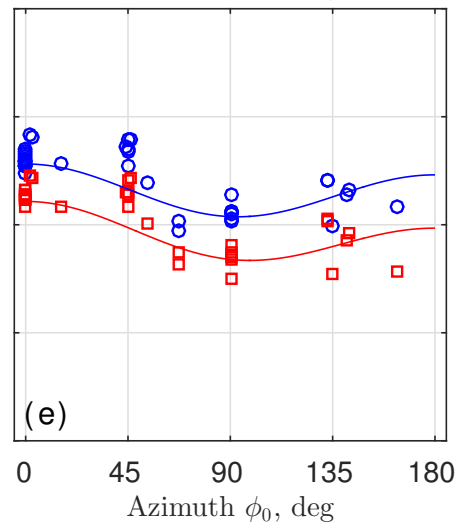

$\theta_{0}=70^{\circ}, U_{0}=8 \mathrm{~m} / \mathrm{s}$
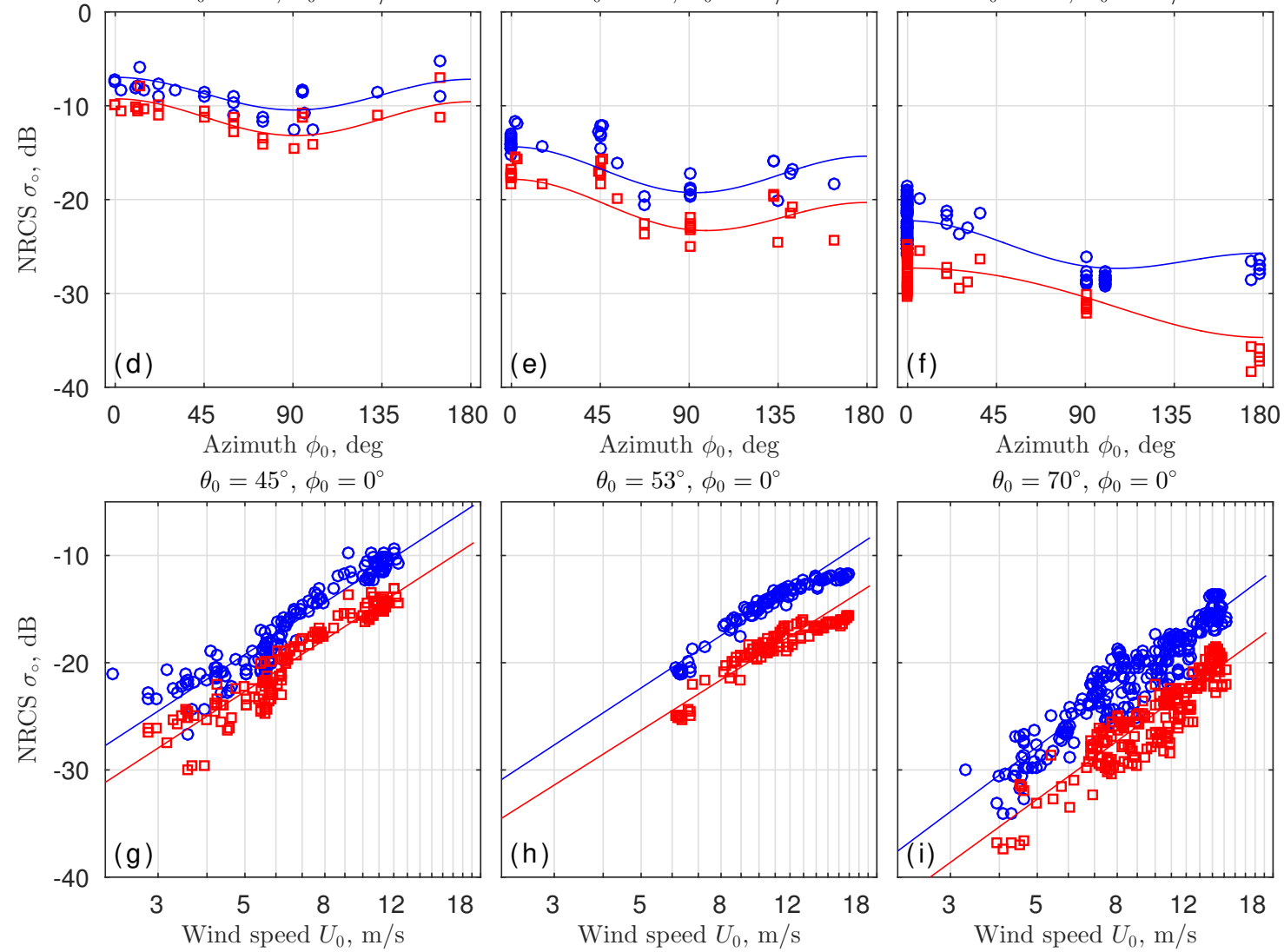

Fig. 5. Measured NRCS versus (a,b,c) incidence angle, $(\mathrm{d}, \mathrm{e}, \mathrm{f})$ azimuth, and (g,h,i) wind speed. Radar-look geometry and wind speed are given in each panel title. Solid lines are the best data fit in form of (6). Blue color is VV polarization, red is HH polarization.

Fig. 5 illustrates an example of measured NRCS as a function of $\theta, \phi$, and $U$ for cases with rather good statistics. The antenna pattern impact on measured NRCS is clearly seen at near-nadir $\theta$, where $\sigma_{\circ}$ for VV polarization is higher than that for HH polarization, while they must be the same for specular reflections dominating at low $\theta$ (Fig. $5 a-c)$. 
In contrast to the incidence angle dependence, the azimuth dependence of measured NRCS is not strongly affected by the antenna pattern, and the "true" NRCS azimuthal features can be directly inferred from measured NRCS. At moderate incidence angles $\left(\theta=45^{\circ}\right.$, Fig. 5e) the azimuth dependence of NRCS has expected characteristics with a minimum in the crosswind direction and local maxima in the upwind and downwind directions. At these angles the upwind backscattering exceeds the downwind backscattering. At lower incidence angles $\left(\theta=32^{\circ}\right.$, Fig. 5d), the upwind minus downwind NRCS difference vanishes (if not actually changes sign) demonstrating that backward wave slopes may be "rougher". At high incidence angles $\left(\theta=70^{\circ}\right.$, Fig. 5f), the azimuth distribution becomes unimodal, with a clear upwind maximum and a minimum in the downwind direction, which is even lower than the crosswind NRCS.

In general, wind dependencies of measured NRCS follow the power law, $\sigma_{\circ} \sim U^{N}$ (Fig. 5g-i). However, some evidence of saturation is seen at rather strong winds, $U>15 \mathrm{~m} / \mathrm{s}$ (Fig. $5 \mathrm{~h}$ ).

As already noticed, the measured NRCS, especially for VV polarization, is significantly affected by the radar antenna pattern. Therefore in order to infer the true NRCS, the measured NRCS must be corrected for the impact of the radar antenna pattern.

\section{RESULTS}

\section{A. Data Fitting}

The effective (measured) NRCS, $\sigma_{\text {oeff }}$, at wind speed, $U$, nominal incidence angle, $\theta_{0}$, and azimuth, $\phi_{0}$, is a convolution of the true sea surface NRCS, $\sigma_{\circ}$, and the radar antenna pattern, $\Gamma_{\mathrm{eff}}$ :

$$
\begin{aligned}
\sigma_{\text {oeff }}\left(\theta_{0}, \phi_{0}, U\right) & =\frac{\int \Gamma_{\text {eff }}(x, y) \sigma_{\circ}(x, y, U) \mathrm{d} x \mathrm{~d} y}{\int \Gamma_{\text {eff }}(x, y) \mathrm{d} x \mathrm{~d} y}= \\
& =\frac{\int \Gamma_{\text {eff }}(\theta, \phi) \sigma_{\circ}(\theta, \phi, U) J(\theta, \phi) \mathrm{d} \theta \mathrm{d} \phi}{\int \Gamma_{\text {eff }}(\theta, \phi) J(\theta, \phi) \mathrm{d} \theta \mathrm{d} \phi},
\end{aligned}
$$

where $J(\theta, \phi)$ is the Jacobian of $\{x, y\}$ - to $\{\theta, \phi\}$-coordinate transformation, $\mathrm{d} x \mathrm{~d} y=J(\theta, \phi) \mathrm{d} \theta \mathrm{d} \phi$ (see Appendix C).

To obtain the true NRCS, $\sigma_{\circ}(\theta, \phi, U)$, from measured NRCS, $\sigma_{\circ \text { off }}\left(\theta_{0}, \phi_{0}, U\right)$, the integral equation (4) is solved numerically by minimizing the norm of residuals between the measured $\sigma_{\text {oeff }}$ and a fitting model for $\sigma_{\circ}$. Following previous studies (see e.g. [13], [27]), the fitting model is represented as a truncated Fourier series:

$$
\sigma_{\circ}=A_{0}(\theta, U)+A_{1}(\theta, U) \cos \phi+A_{2}(\theta, U) \cos 2 \phi
$$

where coefficients, $A_{j}$, are functions of $U$ and $\theta$.

Notice, that Ka-band Bragg waves are strongly affected by viscous dissipation, which in turn depends on sea surface temperature, SST [28]. However, we do not consider SST dependence of the NRCS because our data are collected in a relatively narrow SST range between $18^{\circ} \mathrm{C}$ and $22^{\circ} \mathrm{C}$. 

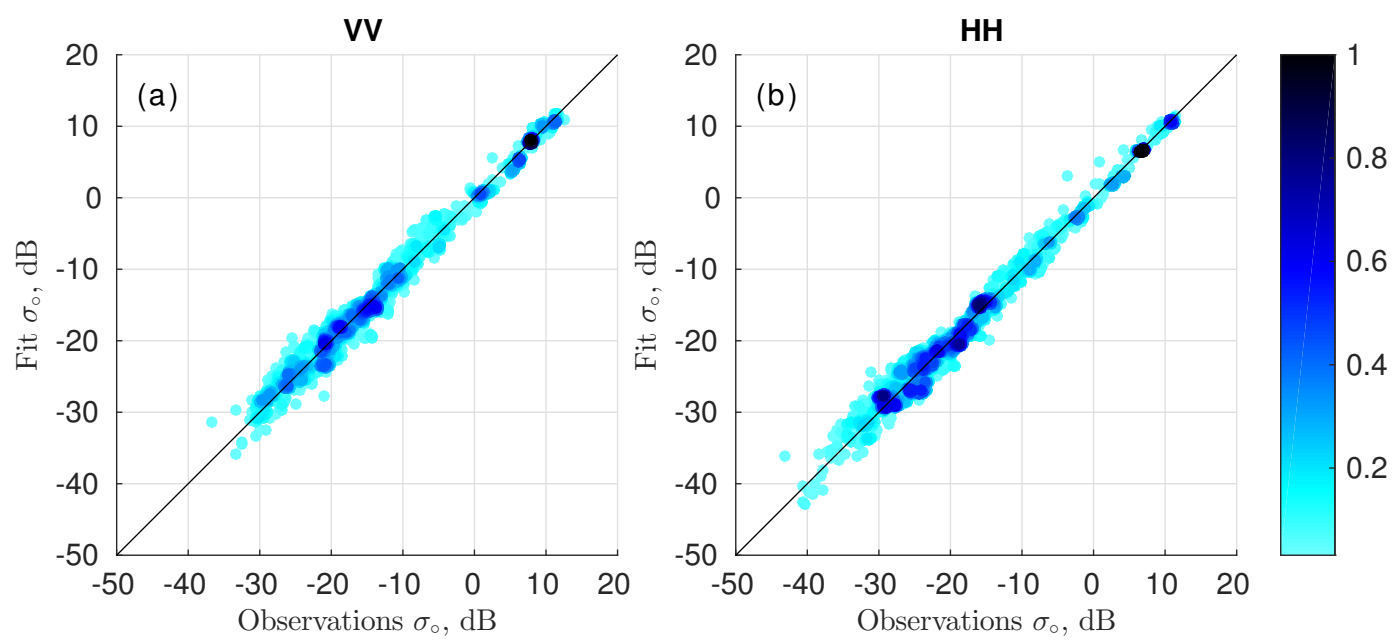

Fig. 6. Scatter diagram of measured NRCS and true NRCS weighted with the radar antenna pattern (see (4)) for (a) VV and (b) HH polarizations. Color indicates the density of data points.

Observed $\sigma_{\text {oeff }}$ varies in a wide range, from $10-15 \mathrm{~dB}$ at near-nadir angles to $-40 \mathrm{~dB}$ (after noise removal) at high incidence angles. For such a large dynamical range, the minimization is more effective for $\sigma_{\circ}$ represented in logarithmic units. Therefore instead of (5), the true NRCS is fitted by the following model:

$$
\log \sigma_{\circ}=A_{0}(\theta, U)+A_{1}(\theta, U) \cos \phi+A_{2}(\theta, U) \cos 2 \phi
$$

with coefficients, $A_{j}$, represented as polynomials of $\theta$ and $\log U$ :

$$
A_{j}=\sum_{m=0}^{4} \sum_{k=0}^{1} C_{m j k} \theta^{m}(\log U)^{k} .
$$

Since the number of measurements is of the order of 1000, the system of linear equations (6) is overdetermined and unknown coefficients, $C_{m n k}$ (30 per each polarization, see Appendix D), are obtained by minimizing the least square error. The true NRCS, $\sigma_{\circ}(\theta, \phi, U)$, satisfies the non-linear integral equation (4), which is solved iteratively using the Nelder-Mead simplex (direct search) method [29] starting from a first guess based on the fitting model (6) for the measured NRCS (shown by solid lines in Fig. 5). Logarithmic scale (6) is used only for data fitting while the resulting empirical fit is transformed back into linear units.

\section{B. Accuracy Testing}

The accuracy of the fitting method is checked by applying the angular antenna pattern to the solution of (6) and comparing the results with the measured NRCS (Fig. 6). The model root-mean-square errors are found to be 1.47 $\mathrm{dB}$ and $1.50 \mathrm{~dB}$ for $\mathrm{VV}$ and $\mathrm{HH}$ polarization, respectively, with 0.98 correlation coefficient.

The limits of applicability of our empirical model (hereinafter, Ka-band Dual co-Polarized Model, or, KaDPMod) are defined by the range of observed conditions and technical limitations of the radar. The minimum detectable wind 
speed at moderate incidence angles is about $3 \mathrm{~m} / \mathrm{s}$ and is determined by the radar noise. The maximum observed wind speed is $18 \mathrm{~m} / \mathrm{s}$. Since the fit is less confident at low incidence angles (due to poor statistics), we suggest using KaDPMod at $25^{\circ}<\theta<65^{\circ}$, where the upper incidence angle limit is also imposed by the radar noise.

\section{The "True" NRCS}

Because the KaDPMod does not depend on the technical characteristics of our particular radar, it can be compared with independent Ka-band data [15], [20], [21] as well as with the Ku-band co-polarized backscatter NSCAT-4 model [30] (Fig. 7, 8, 9).

In general, KaDPMod is systematically higher by $5-10 \mathrm{~dB}$ than MOSN'86 (see also discussions in [17], [18]). But, the KaDPMod agrees well with Plant et al's. co-polarized data [20] collected in the near-upwind direction at $\theta=45^{\circ}$ (Fig. 9b). Giovanageli et al's. [21] laboratory VV measurements are available at $\theta=30^{\circ}$ for all azimuths. The KaDPMod agrees well with [21] in the upwind direction (Fig. 9a), but is $2-4 \mathrm{~dB}$ lower in the downwind and crosswind directions (not shown).

Comparison of KaDPMod with the Ku-band NSCAT-4 model reveals quite reasonable correspondence at high incidence angles, $\theta>45^{\circ}$. However, the KaDPMod significantly exceeds the NSCAT-4 at lower incidence angles (Fig. 7). In particular, the difference reaches $5 \mathrm{~dB}$ at $\theta=30^{\circ}$ and $U=5 \mathrm{~m} / \mathrm{s}$ in the downwind direction (Fig. 7c). Both, KaDPMod and NSCAT-4 models have similar azimuth dependence at rather strong winds, $U \geq 10 \mathrm{~m} / \mathrm{s}$ (Fig. 8). But at weaker winds, $U=5 \mathrm{~m} / \mathrm{s}$, the azimuth dependence of Ka-band NRCS becomes stronger than that in the Ku-band.

At low incidence angles, $\theta<30^{\circ}$, the upwind-downwind difference is near zero or negative (in dB, Fig. 10a-c). At larger incidence angles, this difference grows up to more expected positive values and even becomes stronger than that in Ku-band for both polarizations.

In general, the Ka-band upwind-crosswind difference is similar to that in the Ku-band. It has maximum values at moderate incidence angles, $40^{\circ}<\theta<50^{\circ}$. However at large incidence angles, $\theta \sim 60^{\circ}$, the crosswind Ka-band HH NRCS becomes higher than that in the downwind direction and causes a unimodal azimuth distribution (Fig. 8 and 10). Except at low winds, both KaDPMod and MOSN'86 predict consistent values of upwind-downwind and upwind-crosswind NRCS difference.

\section{Dual Co-polarized Features}

The KaDPMod is further analyzed in terms of the NRCS decomposition into the polarized Bragg component (described by the Two-Scale Model, TSM) and non-polarized (scalar, NP) components. Following [31], the NRCS (in linear units) is represented as:

$$
\sigma_{\circ}^{\mathrm{pp}}=\sigma_{\mathrm{br}}^{\mathrm{pp}}+\sigma_{\mathrm{np}}
$$

where the superscript (Pp) stands for polarization. The NP component is associated with quasi-specular reflection from steep breaking waves and regular (non-breaking) surface. The latter is important at small incidence angles, 


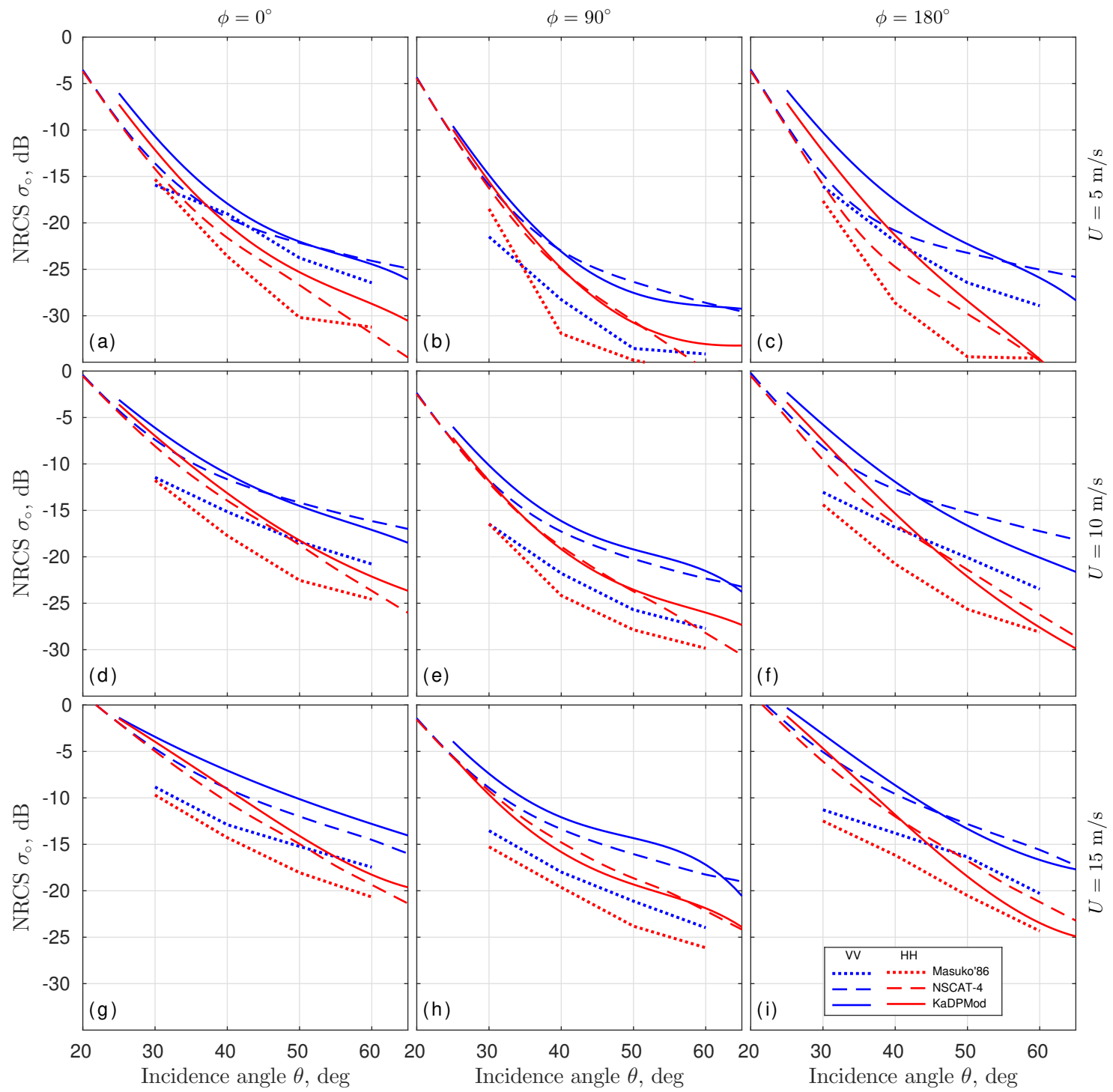

Fig. 7. Dependence of NRCS on incidence angle: (left column) upwind, (middle column) crosswind, (right column) downwind directions. Wind speed is $5 \mathrm{~m} / \mathrm{s}, 10 \mathrm{~m} / \mathrm{s}$, and $15 \mathrm{~m} / \mathrm{s}$ from top to bottom row correspondingly. Blue color - VV polarization, red color - HH polarization. KaDPMod is solid, Ku-band NSCAT-4 is dashed, MOSN'86 is dotted.

$\theta<20^{\circ}$, but in the Ka-band (in contrast to the C- and Ku-bands) it could contribute to the NRCS at larger $\theta$ due to a wider spectral interval of long waves (LW) tilting the Bragg waves. Given known VV and HH NRCS, the NP term, $\sigma_{\mathrm{np}}$, can be estimated from a combination of the TSM polarization ratio (PR, $\left.P_{\mathrm{br}}\right)$ and observed polarization difference (PD, $\Delta \sigma)$ : 


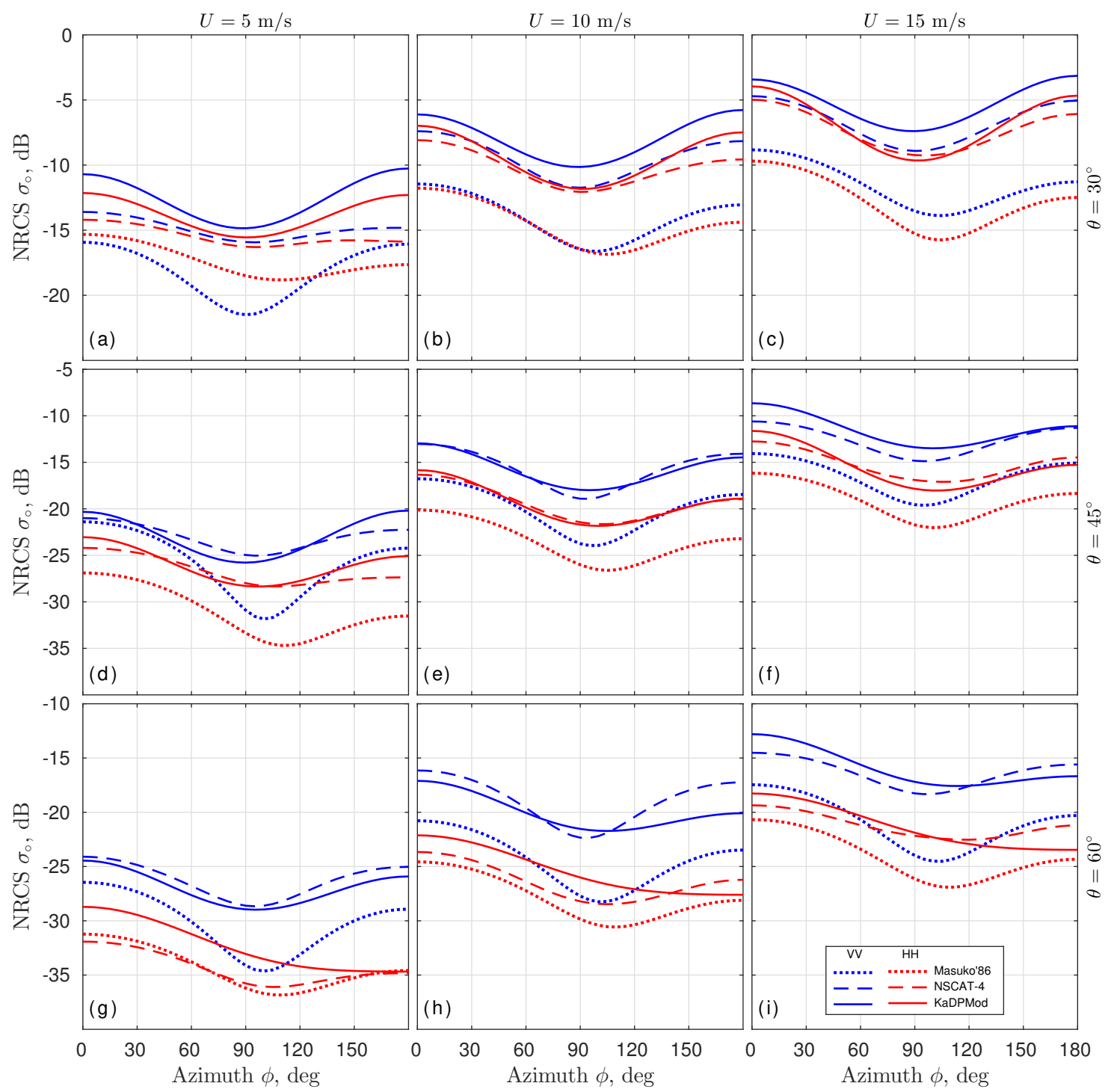

Fig. 8. Azimuth dependence of NRCS at wind speed $5 \mathrm{~m} / \mathrm{s}$ (left column), $10 \mathrm{~m} / \mathrm{s}$ (middle column) and $15 \mathrm{~m} / \mathrm{s}$ (right column). Incidence angle is $30^{\circ}, 45^{\circ}$, and $60^{\circ}$ from top to bottom row correspondingly. Colors and line styles are the same as in Fig. 7.

$$
\begin{aligned}
\sigma_{\mathrm{np}} & =\sigma_{\circ}^{\mathrm{vv}}-\frac{\Delta \sigma}{1-P_{\mathrm{br}}^{-1}}, \\
P_{\mathrm{br}} & =\sigma_{\mathrm{br}}^{\mathrm{vv}} / \sigma_{\mathrm{br}}^{\mathrm{hh}}, \\
\Delta \sigma & \equiv \sigma_{\circ}^{\mathrm{vv}}-\sigma_{\circ}^{\mathrm{hh}}=\sigma_{\mathrm{br}}^{\mathrm{vv}}-\sigma_{\mathrm{br}}^{\mathrm{hh}} .
\end{aligned}
$$

Anticipating that Ka-band scattering is quite complicated and significantly affected by the LW (via tilting, hydrodynamic modulations, and non-linearity of the surface slopes, etc.), we restrict our analysis to moderate incidence angles, $\theta>30^{\circ}$. Then the TSM solution can be represented as the first two terms of a Taylor expansion 

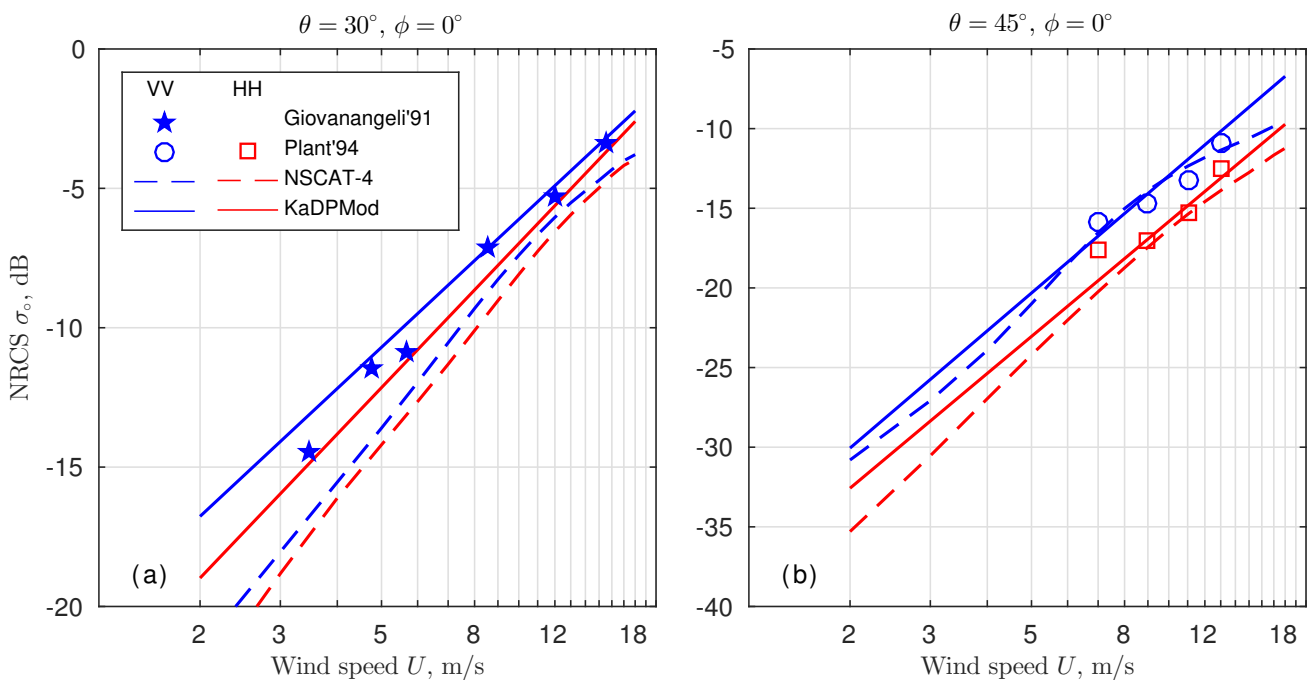

Fig. 9. Wind speed dependence of upwind NRCS at (a) $\theta=30^{\circ}$, (b) $\theta=45^{\circ}$. Colors and line styles are the same as in Fig. 7.

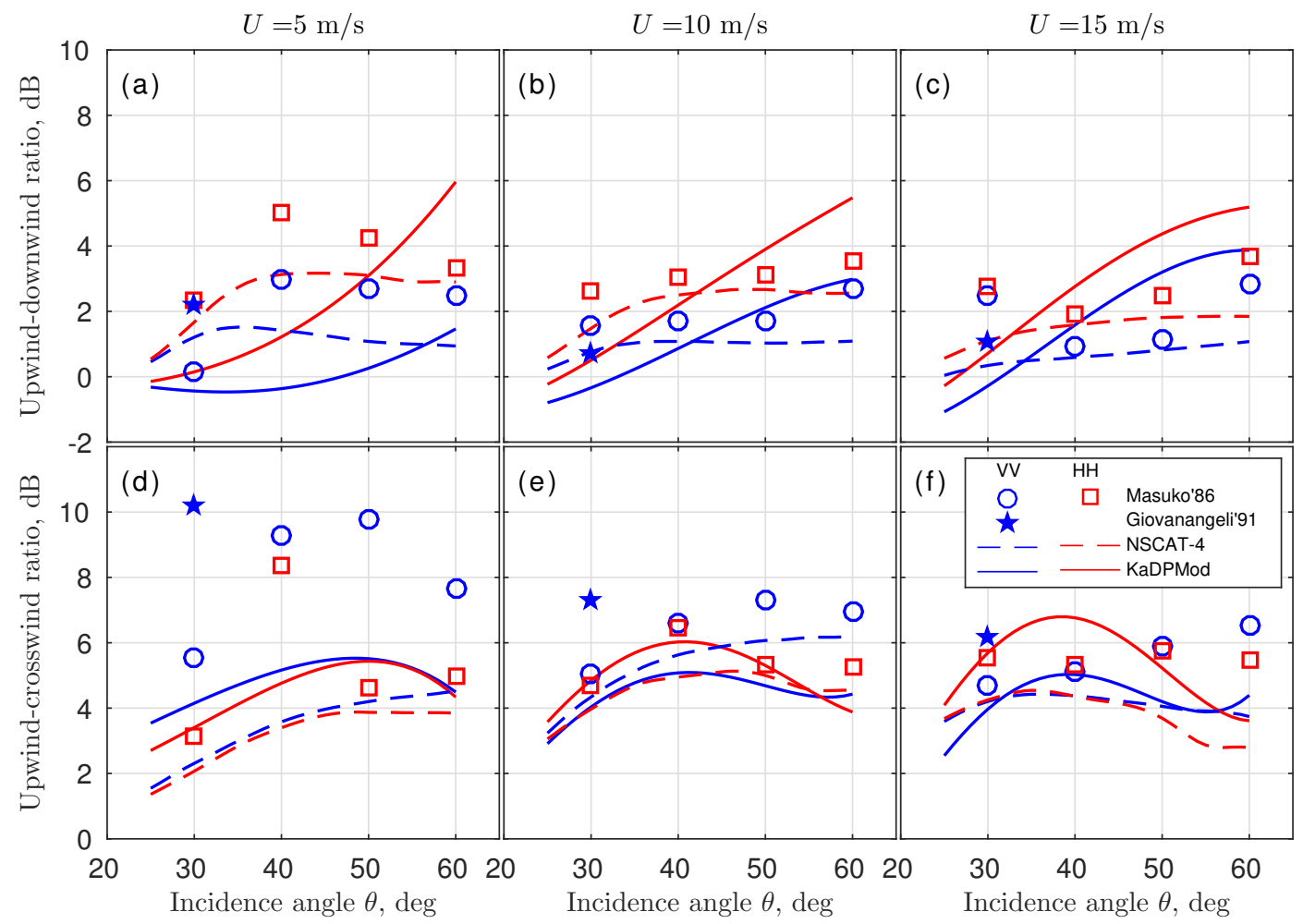

Fig. 10. (a,b,c) Upwind-downwind and (d,e,f) upwind-crosswind NRCS ratio at $U=5 \mathrm{~m} / \mathrm{s}, 10 \mathrm{~m} / \mathrm{s}$ and $15 \mathrm{~m} / \mathrm{s}$ versus incidence angle. Colors and linestyles are the same as in Fig. 7. 
in LW slope (see e.g. [32], [33]):

$$
\sigma_{\mathrm{br}}^{\mathrm{pp}}=\pi G_{\mathrm{pp}}^{2} B\left(\mathbf{k}_{\mathrm{br}}\right)\left(1+g_{\mathrm{pp}} \overline{\zeta_{\mathrm{i}}^{2}}-\frac{M_{\mathrm{t}}^{\mathrm{pp}}}{B} \overline{\zeta_{\mathrm{i}} \tilde{B}}\right)
$$

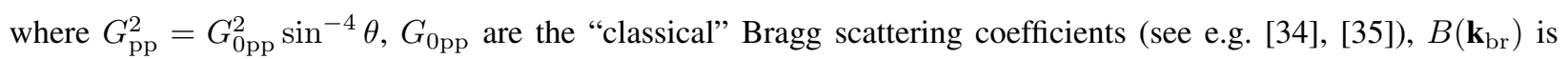
the folded saturation spectrum at Bragg wavenumber $k_{\mathrm{br}}=2 k_{\mathrm{r}} \sin \theta, k_{\mathrm{r}}$ is the radar wavenumber, $\tilde{B}$ is the Bragg spectrum variation due to the $\mathrm{LW}, \overline{\zeta_{\mathrm{i}}^{2}}$ is the $\mathrm{LW}$ mean-square slope in the incidence plane direction, and the tilt modulation transfer function (MTF) $M_{\mathrm{t}}^{\mathrm{pp}}$ is

$$
M_{\mathrm{t}}^{\mathrm{pp}}=G_{\mathrm{pp}}^{-2} \frac{\partial G_{\mathrm{pp}}^{2}}{\partial \theta}
$$

The geometric coefficients $g_{\mathrm{pp}}$ in (12) are

$$
\begin{aligned}
& g_{\mathrm{vv}}=\frac{1}{2 G_{\mathrm{vv}}^{2}} \frac{\partial^{2} G_{\mathrm{vv}}^{2}}{\partial \theta^{2}}, \\
& g_{\mathrm{hh}}=\frac{1}{2 G_{\mathrm{hh}}^{2}} \frac{\partial^{2} G_{\mathrm{hh}}^{2}}{\partial \theta^{2}}+\frac{2}{\sin ^{2} \theta} \frac{\left|G_{\mathrm{vv}}\right|}{\left|G_{\mathrm{hh}}\right|} \frac{\overline{\zeta_{\mathrm{c}}^{2}}}{\overline{\zeta_{\mathrm{i}}^{2}}},
\end{aligned}
$$

where $\overline{\zeta_{c}^{2}}$ is the LW mean-squared slope in the direction normal to the radar incidence plane.

Representing the Bragg wave modulations via the hydrodynamic MTF, $\tilde{B} / B=M_{\mathrm{h}} k a$, where $a$ is the wave amplitude, and disregarding the spectral dependence of $M_{\mathrm{h}}$, the mean contribution to NRCS in (12) arising from the correlation between the tilt and hydrodynamic modulations can be written as: $\left(M_{\mathrm{t}}^{\mathrm{pp}} / B\right) \overline{\zeta_{\mathrm{i}} \tilde{B}}=-M_{\mathrm{t}}^{\mathrm{pp}} M_{\mathrm{h}}^{\mathrm{I}} \overline{\zeta_{\mathrm{i}}^{2}} \cos \phi$, where $M_{\mathrm{h}}^{\mathrm{I}}$ is the imaginary part of the hydrodynamic MTF, which sign is chosen so that $M_{\mathrm{h}}^{\mathrm{I}}>0$ if the Bragg waves enhance on the forward (downwind) slope of the LW. Then (12) can be rewritten as:

$$
\begin{aligned}
\sigma_{\mathrm{br}}^{\mathrm{pp}} & =\pi G_{\mathrm{pp}}^{2} B\left(\mathbf{k}_{\mathrm{br}}\right)\left(1+g_{\mathrm{pp}} \overline{\zeta_{\mathrm{i}}^{2}}+h_{\mathrm{pp}} \overline{\zeta_{\mathrm{i}}^{2}} \cos \phi\right), \\
h_{\mathrm{pp}} & =M_{\mathrm{t}}^{\mathrm{pp}} M_{\mathrm{h}}^{\mathrm{I}} .
\end{aligned}
$$

\section{A. Polarization Ratio}

The polarization ratio, PR, is a direct indicator of the relative contribution of different backscattering mechanisms. Depending on the relative NP contribution, the PR varies from 1 (if NP scattering dominates) to $P_{\mathrm{br}}$ (if NP scattering is negligible):

$$
P=\frac{\sigma_{\mathrm{br}}^{\mathrm{vv}}+\sigma_{\mathrm{np}}}{\sigma_{\mathrm{br}}^{\mathrm{hh}}+\sigma_{\mathrm{np}}}
$$

The relative roles of the Bragg and NP components are evaluated from observed PR and corresponding TSM PR. The latter is computed without accounting for the hydrodynamic modulations (the last term in (15) is omitted). For the sake of simplicity, the LW mean-square slope is estimated from the Phillips saturation spectrum [36], $\overline{\zeta^{2}}=4.6 \cdot 10^{-3} \ln \left(k_{d} / k_{p}\right) / 2$, where $k_{p}=g / U^{2}$ is the peak wavenumber and $k_{d}=k_{\mathrm{br}} / 4$ is the dividing wavenumber. Note, that TSM PR does not depend on the Bragg wave spectrum, but is a function of $\theta$ and weakly depends on wind speed via the LW slope. 

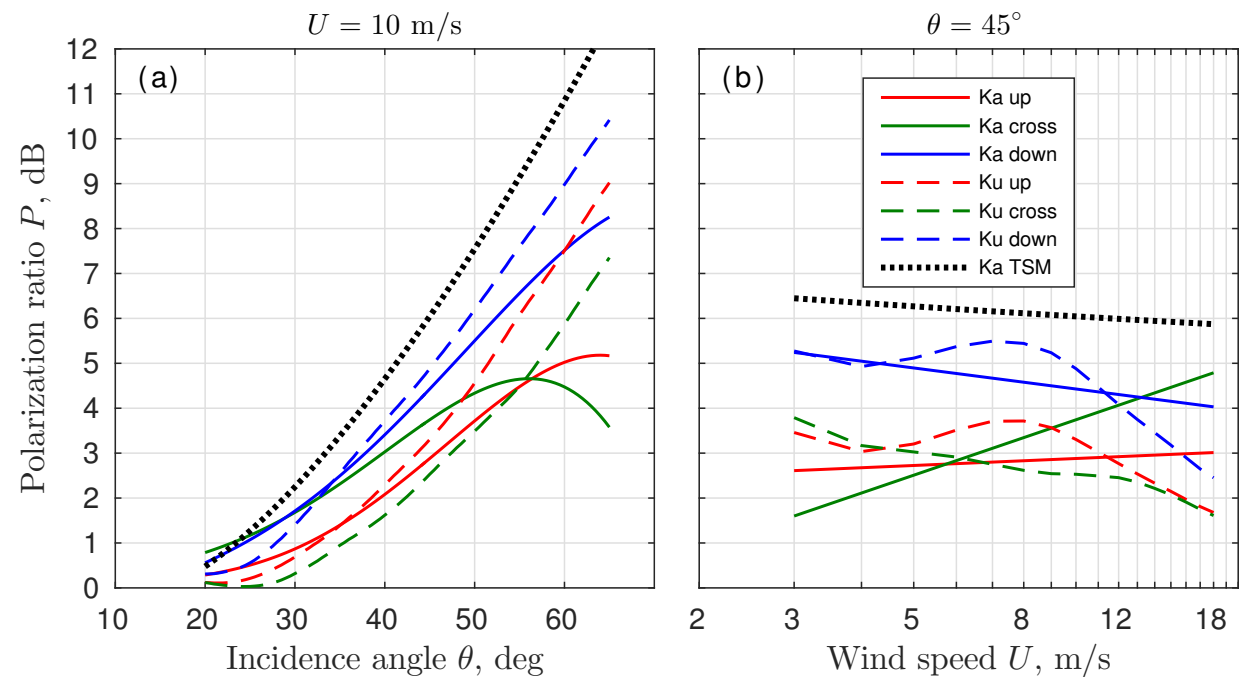

Fig. 11. Polarization ratio as a function of (a) incidence angle at $U=10 \mathrm{~m} / \mathrm{s}$ and (b) wind speed at $\theta=45^{\circ}$ from KaDPMod (solid lines), Ku-band NSCAT-4 (dashed lines) and Bragg Two-Scale Model (TSM, black dotted lines) for upwind (red), crosswind (blue) and downwind (green) directions.

Both Ka- and Ku-band PR are lower than TSM PR indicating a non-negligible NP scattering (Fig. 11). Observed PR is closer to the Bragg TSM PR in the downwind direction where the NP scattering by breaking waves is minimal. At $\theta<55^{\circ}$, the PR is minimal in the upwind direction suggesting that the impact of the NP component is the strongest in this direction. At $\theta>55^{\circ}$, the maximal impact of NP shifts into the crosswind direction where Bragg scattering is weak. In general, the Ka-band PR is qualitatively similar to that in the Ku-band, except for $\theta>45^{\circ}$ where the Ka-band PR is lower due to stronger NP scattering by breaking waves.

The upwind and crosswind PR increase with the wind towards TSM PR (Fig. 11b). This suggests that wind growth rate of capillary Bragg waves is stronger than wind growth rate of wave breaking responsible for NP scattering. Remarkably, this feature distinguishes the Ka-band from the Ku-band (Fig. 11b) and the C-band [37], for which the relative role of the Bragg backscattering weakens as the wind strengthens.

\section{B. Polarization Difference}

By definition (11), the polarization difference (PD) does not include the NP backscattering, and thus it describes only Bragg scattering characteristics. The Bragg waves (and thus PD) are strongly wind dependent (Fig. 12). Both Ka- and Ku-band PD demonstrate strong wind directionality with an apparent minimum in the crosswind direction. The KaDPMod wind exponent is about 2.5 and 2 in the upwind and downwind directions, respectively. The Kuband NSCAT- 4 wind exponent is about 2 in these two directions. In the crosswind direction, the KaDPMod wind exponent $(\sim 3)$ exceeds its upwind and downwind values. This is in contrast to the Ku-band, which has lower crosswind wind exponent $<2$.

The upwind-downwind asymmetry of PD has a more complicated behavior. In the frame of the Bragg TSM, 

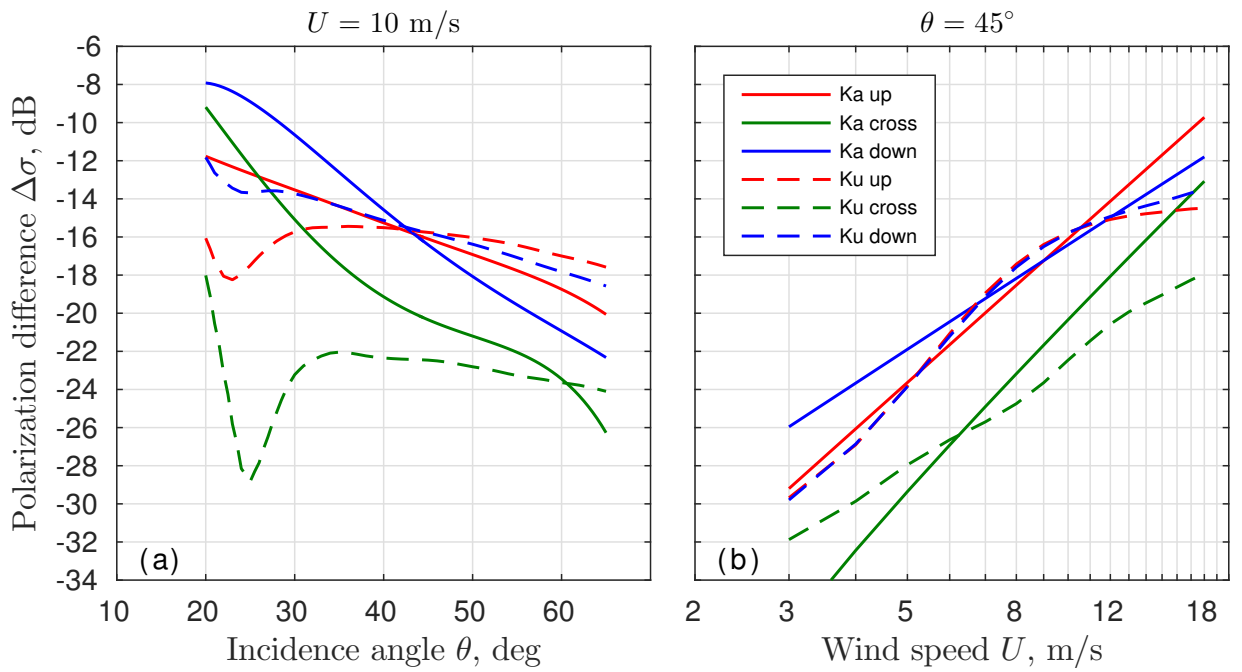

Fig. 12. The same as Fig. 11, but for PD, without TSM prediction.

this asymmetry is attributed to the correlation of Bragg wave energy with the tilting LW slopes (the last term in (15)), which is positive/negative if Bragg roughness enhances on forward/backward slopes, respectively. The Kaand Ku-band models suggest that both signs of upwind-downwind asymmetry are possible. In particular (Fig. 12a), the downwind PD is larger than upwind PD at $\theta<\sim 45^{\circ}$ for $U=10 \mathrm{~m} / \mathrm{s}$, and vice versa at larger incidence angles. Interestingly, that Ka- and Ku-band PD asymmetry both change sign at the same $\theta \approx 43^{\circ}$ for $U=10 \mathrm{~m} / \mathrm{s}$. At fixed $\theta=45^{\circ}$, the Ka-band upwind-downwind PD asymmetry is wind dependent (Fig. 12b). At $U \approx 10 \mathrm{~m} / \mathrm{s}$, the KaDPMod downwind PD is larger than upwind PD, but this asymmetry changes sign at stronger winds. However at $\theta=45^{\circ}$, the Ku-band upwind and downwind PD are almost identical.

The presence of Ka-band PD absolute maximum in the upwind direction at high winds and large incidence angles (Fig.12) is expected due to the effect of enhancement of the parasitic capillary (bound) waves on the forward slopes of the LW. But, the origin of the downwind absolute maximum of PD, which is observed at lower winds and smaller incidence angles in the Ka-band and Ku-band as well as in the C-band [24], is not clear. Here we may only speculate that it is probably caused by the small scale roughness covering the backward slopes of breaking waves, which dominates the upwind-downwind asymmetry at low incidence angles, $\theta<45^{\circ}$. At the larger incidence angles, the Bragg roughness (including parasitic capillaries) covering the forward breaking wave slope, which is steeper than backward, dominates the asymmetry.

\section{Bragg Wave Spectrum Retrieval}

Following the Bragg TSM paradigm, the Bragg wave spectrum, $B\left(\mathbf{k}_{\mathrm{br}}\right)$, can be estimated from the observed PD using (15) rewritten as:

$$
\Delta \sigma=\pi B\left(\mathbf{k}_{\mathrm{br}}\right)\left[\Delta\left(s_{\mathrm{pp}}\right)+\Delta\left(G_{\mathrm{pp}}^{2} h_{\mathrm{pp}}\right) \overline{\zeta^{2}} \cos \phi\right]
$$



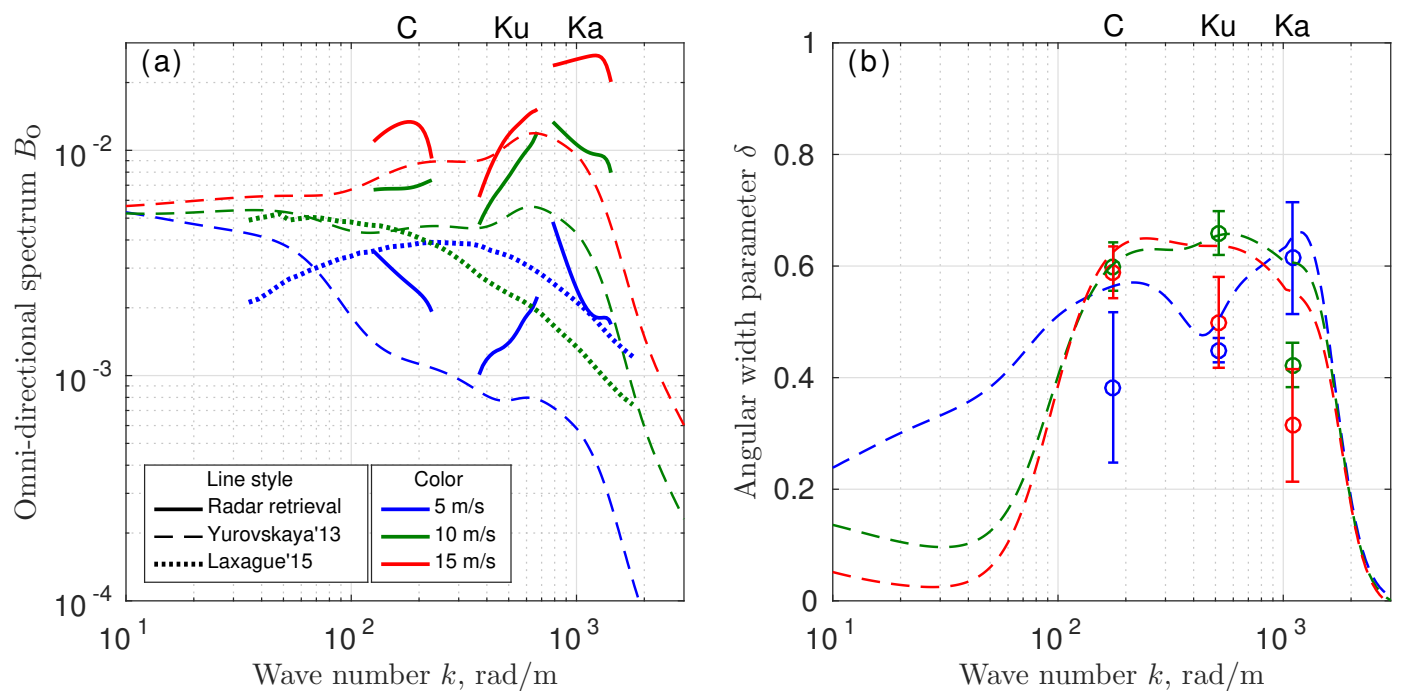

Fig. 13. (a) Omni-directional saturation spectra, $B_{\mathrm{O}}$, and (b) angular width parameter, $\delta$, estimated from radar polarization difference for $\mathrm{C}$ (C-SARMOD), Ku- (NSCAT-4) and Ka-bands (KaDPMod) in comparison with optical measurements [38], [39].

where $s_{\mathrm{pp}}=G_{\mathrm{pp}}^{2}\left(1+g_{\mathrm{pp}} \overline{\zeta^{2}}\right)$, and $\Delta\left(f_{\mathrm{pp}}\right)=f^{\mathrm{vv}}-f^{\mathrm{hh}}$. Representing the Bragg wave saturation spectrum in the same way as in [38]:

$$
B\left(\mathbf{k}_{\mathrm{br}}\right)=\frac{1}{2 \pi} B_{\mathrm{o}}\left(k_{\mathrm{br}}\right)(1+\delta \cos 2 \phi),
$$

where $B_{\mathrm{o}}\left(k_{\mathrm{br}}\right)=\int_{0}^{2 \pi} B\left(k_{\mathrm{br}}, \phi\right) \mathrm{d} \phi$ is the omni-directional saturation spectrum and $\delta$ is the angular width parameter, and using (5) and (19) we have:

$$
\begin{aligned}
\Delta \sigma & \approx A_{0}^{\mathrm{PD}}+A_{1}^{\mathrm{PD}} \cos \phi+A_{2}^{\mathrm{PD}} \cos 2 \phi \\
A_{0}^{\mathrm{PD}} & =B_{\mathrm{o}}\left(k_{\mathrm{br}}\right) \Delta\left(s_{\mathrm{pp}}\right) / 2, \\
A_{1}^{\mathrm{PD}} & =B_{\mathrm{o}}\left(k_{\mathrm{br}}\right) \Delta\left(G_{\mathrm{pp}}^{2} h_{\mathrm{pp}}\right) \overline{\zeta^{2}} / 2, \\
A_{2}^{\mathrm{PD}} & =B_{\mathrm{o}}\left(k_{\mathrm{br}}\right) \Delta\left(s_{\mathrm{pp}}\right) \delta / 2 .
\end{aligned}
$$

The coefficients, $A_{j}^{\mathrm{PD}}$, in (20) are estimated from observations (see Appendix D, with $\Delta \sigma$ instead of $\sigma_{\circ}$ in (40-42)). The omni-directional spectrum and the angular width parameter are calculated using $(16,21-23)$ and observation-based coefficients, $A_{j}^{\mathrm{PD}}$ :

$$
\begin{aligned}
B_{\mathrm{o}}\left(k_{\mathrm{br}}\right) & =2 A_{0}^{\mathrm{PD}} / \Delta\left(s_{\mathrm{pp}}\right), \\
\delta & =A_{2}^{\mathrm{PD}} / A_{0}^{\mathrm{PD}} .
\end{aligned}
$$

The estimates of $B_{\mathrm{o}}$ and $\delta$ retrieved from the Ka-band PD as well as from the Ku-band NSCAT- 4 and C-band C-SARMOD [24] are compared with optically-based field measurements [38], [39] (Fig. 13). 
Radar-derived wave spectra demonstrate stronger wind dependence and higher levels in the capillary range. The latter suggests that the parasitic capillary (bound) mechanism plays a crucial role in establishing of the spectral level of capillary waves. Although radar and optical spectra are very roughly similar in spectral level, the radar spectra are about two times higher. The possible origin of such a difference is in the way the two kind of spectra are derived. Optical spectra are normally derived from wave breaking-free images while radar spectra account for the entire sea surface backscattering including rough patterns of breaking waves. Therefore, the difference between the radar and the optical spectra can be treated as an impact of surface roughness covering wave breaking zones. Optical spectra from [38] and our radar spectra clearly indicate the presence of a peak in the saturation spectra in the capillary range around $k=1000 \mathrm{rad} / \mathrm{m}$ originating from the generation of parasitic capillaries. In that respect, our measurements deviate from recent optical polarimetric measurements [39], which show a decrease in spectral level at wavenumbers above $k=360 \mathrm{rad} / \mathrm{m}$.

The angular width of radar spectra, $\delta \approx 0.5$, indicates that Bragg crosswind wave energy is about 3 times lower than wave energy in the downwind direction, which qualitatively agrees with optical measurements [38] (Fig. 13b). The angular distribution of radar spectra is weakly wind dependent. It is quite narrow in the capillary and capillary-gravity range and becomes much wider in the short gravity wave range.

\section{Non-Polarized Contribution}

Ka- and Ku-band NP components of NRCS, $\sigma_{\mathrm{np}}$, estimated from corresponding model functions using (9) show general consistency in magnitude as well as their dependency on wind speed and incidence angle (Fig. 14). Nevertheless, some important differences in their azimuth variations are present. While the azimuth distribution of Ku-band NP backscattering is unimodal (with a maximum in the upwind direction), the Ka-band azimuth distribution is bimodal at $\theta<50^{\circ}$ (with a minimum in the crosswind direction) and becomes unimodal only at larger incidence angles (Fig. 14a). Such unimodal azimuth distribution of $\sigma_{\mathrm{np}}$ can occur if the NP is dominated by radar return from the forward slope of breaking waves. This is plausible at large incidence angles when the local incidence angle on the backward wave slope is rather large and corresponding backscattering is weak. At moderate incidence angles, a bimodal azimuth distribution of NP backscattering is feasible if the NP from "enhanced" roughness on the backward slope of breaking waves becomes comparable with NP backscattering from the forward slopes.

Wind exponent of the Ka-band NP component is close to 2 (Fig. 14b). This wind exponent is in contrast to the cubic wind dependence of whitecap areal coverage usually considered as an indicator of wave breaking (e.g. [40]). However, the sea surface radar backscattering is sensitive to very different parameters of wave breaking, which are not directly associated with the whitecap areal coverage. One may speculate that NP backscattering is produced by quasi-specular reflections from steep patterns covering breaking wave crests with wavelengths of the order of decimeter and longer scales. These waves belong to the equilibrium range where the wave breaking parameters are proportional to $U^{2}$ (see [33], [41] for more discussions).

The relative contribution of upwind and downwind NP backscattering to the total NRCS has a similar magnitude in the Ka- and Ku-bands at $\theta>40^{\circ}$ (Fig. 15). At HH polarization, the NP contribution to NRCS becomes dominant 

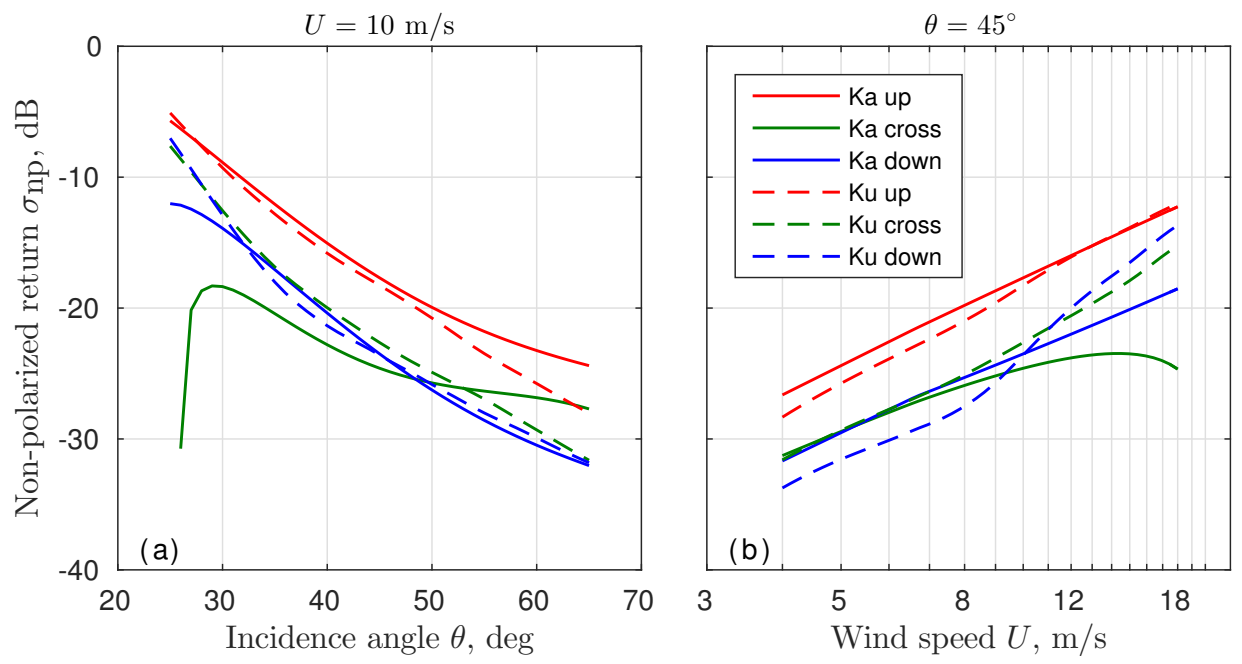

Fig. 14. Non-polarized NRCS component versus (a) incidence angle, (b) wind speed for upwind (red), crosswind (green) and downwind (blue) directions from KaDPMod (solid) and Ku-band NSCAT-4 (dashed).
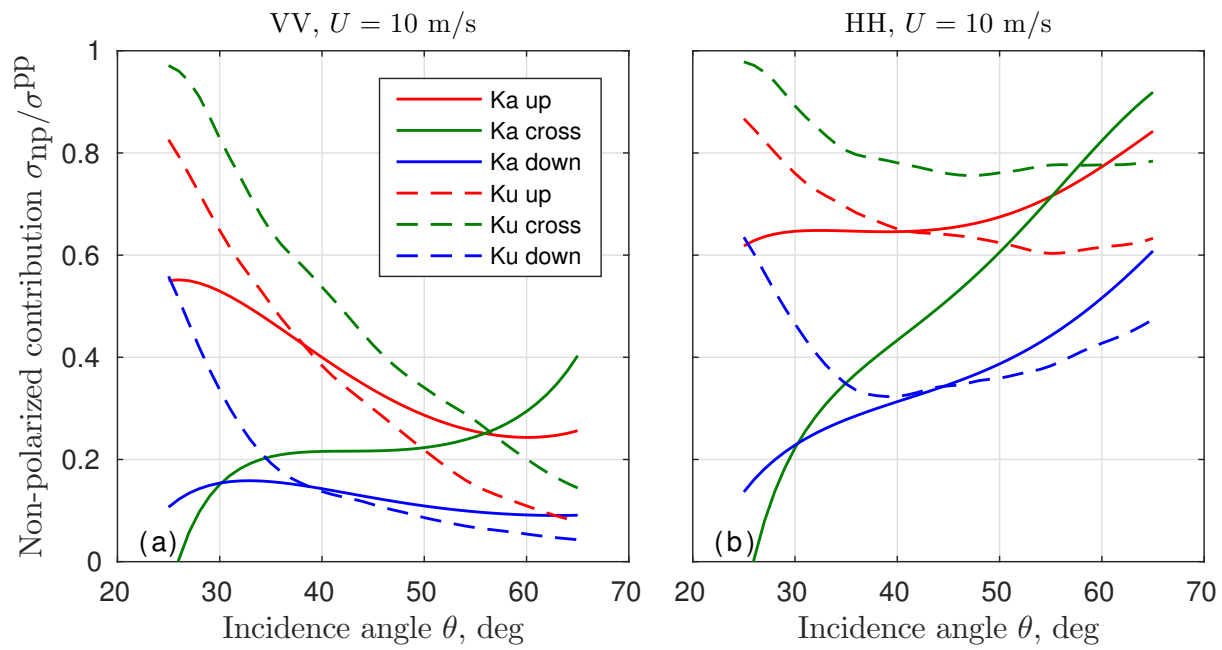

Fig. 15. Partial contribution of non-polarized radar backscattering to total NRCS versus incidence angle for (a) VV and (b) HH polarization. Colors and line styles are the same as in Fig. 14.

in both bands as $\theta$ increases (Fig. 15b). At smaller incidence angles, $\theta<40^{\circ}$, the relative contribution of the NP is lower in Ka-band in comparison with the Ku-band. Because the saturation spectrum level is higher at the Ka-band Bragg wavenumber (Fig. 13a), comparable magnitudes of NP backscattering provide a relatively smaller contribution to the Ka-band NRCS in comparison to the Ku-band.

The observed decrease of the relative contribution of the NP to Ka-band NRCS with increasing wind speed is rather unexpected. However, Fig. 12b and Fig. 14 suggest that Bragg waves (associated with the PD) grow faster 

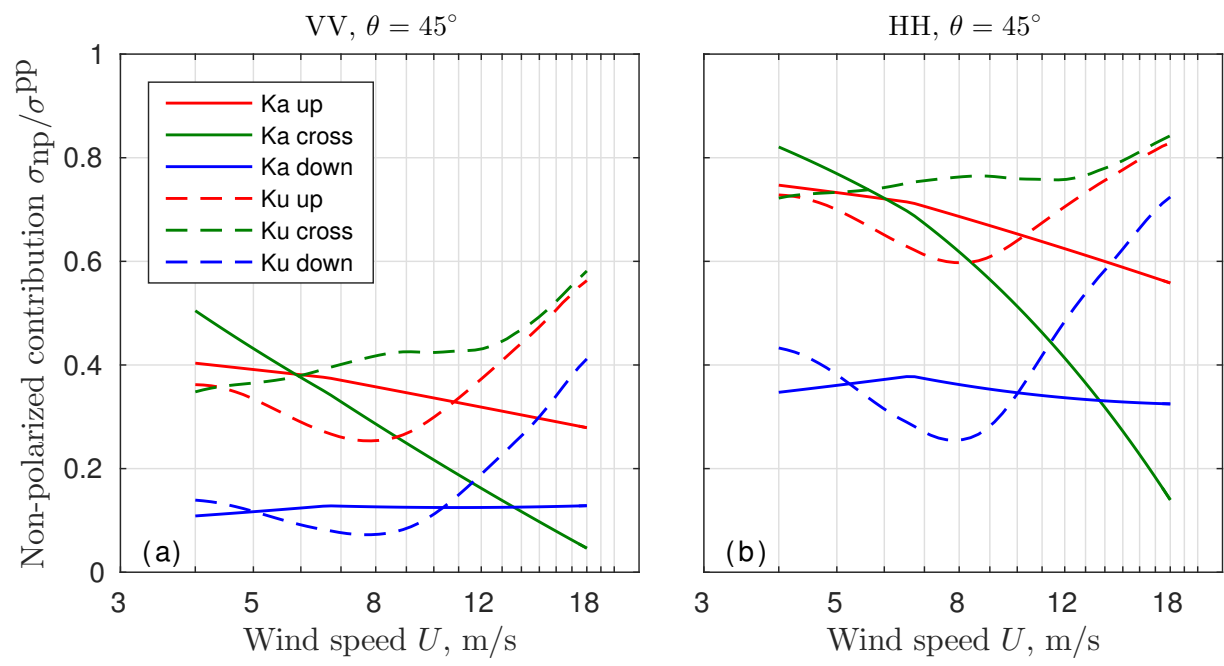

Fig. 16. The same as Fig. 15, but versus wind speed.

with the wind than the NP term. Therefore, the relative contribution of NP backscattering to the NRCS should decrease with the wind. This behavior is in line with observations in Fig. 16.

\section{SUMmary}

In this paper we report the Ka-band dual co-polarized ( $\mathrm{VV}$ and $\mathrm{HH}$ ) backscattering features of the sea surface derived from measurements collected from the Black Sea Research platform during seven years of field campaigns between 2009-2015. The radar measurements are collected in the incidence angles range: $25^{\circ}<\theta<65^{\circ}$, the wind speed range: $3 \mathrm{~m} / \mathrm{s}<U<18 \mathrm{~m} / \mathrm{s}$, and with the radar-to-wind azimuth varying from upwind to downwind. The radar measurements are corrected for the impact of the antenna angular pattern and are presented in a form of a conventional truncated azimuthal Fourier series with coefficients dependent on incidence angle and wind speed. This parameterization is referred as an empirical Ka-band Dual co-Polarized radar backscattering Model (KaDPMod). We anticipate that this empirical model can be used as a first guess for developing of Ka-band geophysical model function for the sea surface NRCS.

To the best of our knowledge, KaDPMod is the first attempt to parametrize the Ka-band sea surface NRCS at moderate incidence angles for both VV and HH polarizations since the MOSN'86 model [15] and its re-evaluation [16]. We find that KaDPMod are 5-10 dB higher than the MOSN' 86 data, which is believed to be due (after [17]) to uncertainties in their data calibration. KaDPMod is consistent with independent Ka-band data collected in the field [20] and also under laboratory conditions [21]. In general, KaDPMod is consistent with the empirical Ku-band NSCAT-4 model over a wide range of wind speeds and incidence angles. However, there are some remarkable differences between the $\mathrm{Ku}-$ and Ka-band models.

To get deeper insight into the physics of Ka-band sea surface backscattering the dual co-polarized measurements are decomposed into resonant Bragg backscattering and non-polarized (NP) backscattering from breaking waves [31]. 
The relative importance of NP is confirmed by the presence of a significant deviation of the measured polarization ratio (PR, $\sigma_{\circ}^{\mathrm{vv}} / \sigma_{\circ}^{\mathrm{hh}}$ ) from the Bragg Two-Scale Model (TSM). Unlike C- and Ku-bands, the Ka-band PR increases strongly with wind towards the TSM PR values. This suggests that the wind-induced growth of Bragg waves is stronger than that of NP backscattering associated with wave breaking.

The polarization difference ( $\mathrm{PD}, \sigma_{\circ}^{\mathrm{vv}}-\sigma_{\circ}^{\mathrm{hh}}$ ) allows us to eliminate the NP component and provides a direct information about short Bragg waves. Our measurements show that PD, and thus Ka-band Bragg waves, is strongly wind dependent, with a wind exponent of about 2.5 and 3 in the upwind and crosswind direction, respectively. That is consistent with optical measurements of wave spectra [38], [42]. The PD (and thus capillary Bragg waves) is strongly anisotropic in azimuth with a minimum in the crosswind direction. Upwind-to-crosswind anisotropy is quantitatively consistent with independently measured spectra in the capillary range [38]. But, the upwind-todownwind asymmetry has a more complex behavior. At $\theta<45^{\circ}$ and $U<10 \mathrm{~m} / \mathrm{s}$, the downwind PD exceeds the upwind PD, suggesting that Ka-band Bragg waves are enhanced on the backward slopes of long tilting waves. At larger incidence angles and stronger winds the PD maximum shifts towards the upwind direction that is anticipated if Bragg waves are mainly generated as parasitic capillaries.

Omni-directional wave spectra derived from the PD based on KaDPMod, Ku-band NSCAT-4 [30] and C-band CSARMOD [24] models, provide a quite consistent description of short wind wave spectra, and indicate a noticeable peak in the saturation spectrum level in the capillary-gravity range. This observation is in contrast to the recent experimental finding [39] suggesting a "slight" roughness contribution from capillary waves and a significant contribution from gravity-capillary waves.

Ka-band non-polarized (NP) NRCS component derived from KaDPMod is similar to the Ku-band NP derived from the NSCAT-4 empirical model. The relative contribution of the NP component to the total NRCS is significant. In the upwind direction, it is about $60 \%-80 \%$ for $\mathrm{HH}$ polarization, and about $25 \%-50 \%$ for VV polarization. The NP wind exponent is about 2 in the upwind direction, which is lower than the wind exponent for Ka-band Bragg waves. This explains the decrease in the relative contribution of NP component to the NRCS for both polarizations. Such wind dependence of the relative NP contribution is opposite to that suggested by the Ku-band NSCAT-4, for which the relative contribution of NP to the total NRCS increases with the wind.

\section{APPENDIX A}

\section{RADAR CALIBRATION}

The radar pattern is crucial for radar calibration and estimation of the effective footprint area. The two-way radar angular antenna pattern is estimated using a metal $100 \mathrm{~mm}$ sphere target (installed on thin dielectric lines in front of the radar directed towards the clear sky) and video camera synchronized with the radar (Fig. 17).

Oscillations of the target are synchronously registered by radar and video camera, thus yielding the radar backscattering as a function of the target's angular coordinates, $\alpha$ and $\beta$. The two-way radar pattern $\Gamma$ is then estimated as the distribution of the received power versus $\alpha$ and $\beta$ normalized by the peak value (Fig. 18).

The calibration constant, $C$, in (1) is estimated from observations of different targets at known distances and 

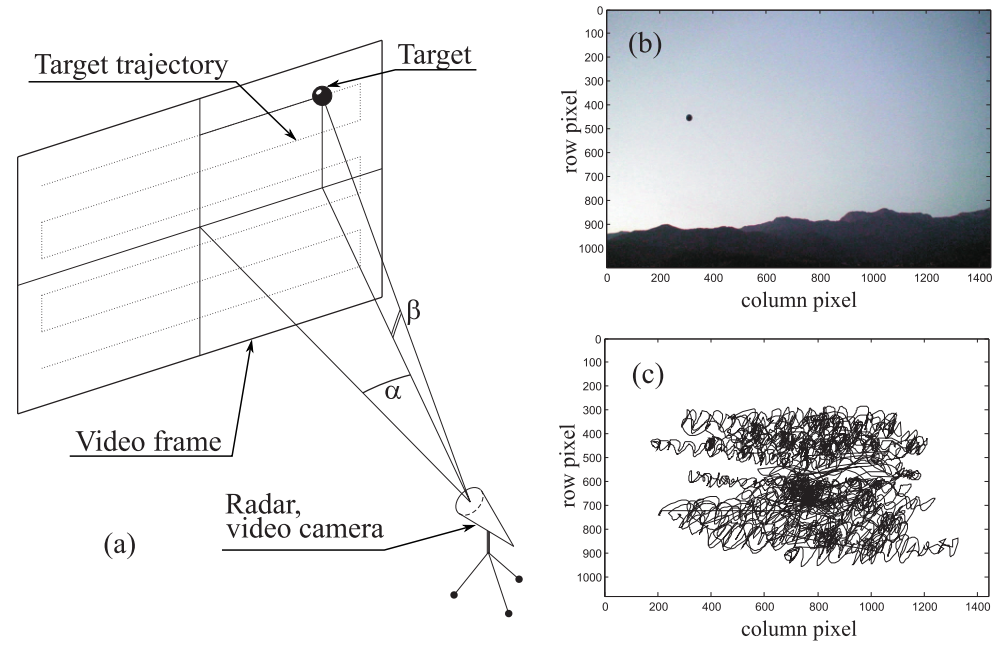

Fig. 17. Radar calibration: (a) schematic explanation of the method, (b) target video image on the background of clear skies (mountains at the image bottom are tens of kilometers apart), (c) sample target trajectory.
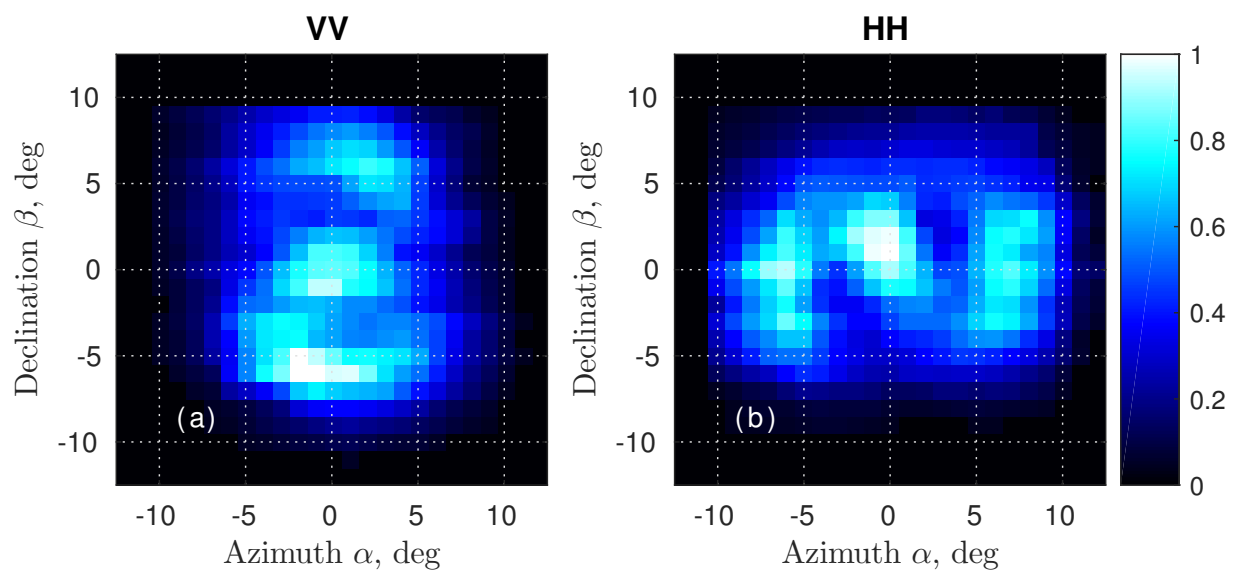

Fig. 18. Normalized two-way radar patterns (a) $\Gamma^{\mathrm{hh}}$ and (b) $\Gamma^{\mathrm{vv}}$.

positions within the radar pattern. The relationship between the power received by the radar and the recorded signal power is determined by characteristics of the radar hardware, which may introduce some non-linearity. For calibration purposes, the received power was varied by changing target type, size, and distance. The relationship between the radar output signal variance and received power is found to be linear in the whole range of signal amplitudes and is used to determine the calibration constant, $C$ (Fig. 19a). One can note very close signal levels in both channels (Fig. 19b, except for the smallest calibration target with bad signal recognition) suggesting that vertical and horizontal channels are well balanced. 

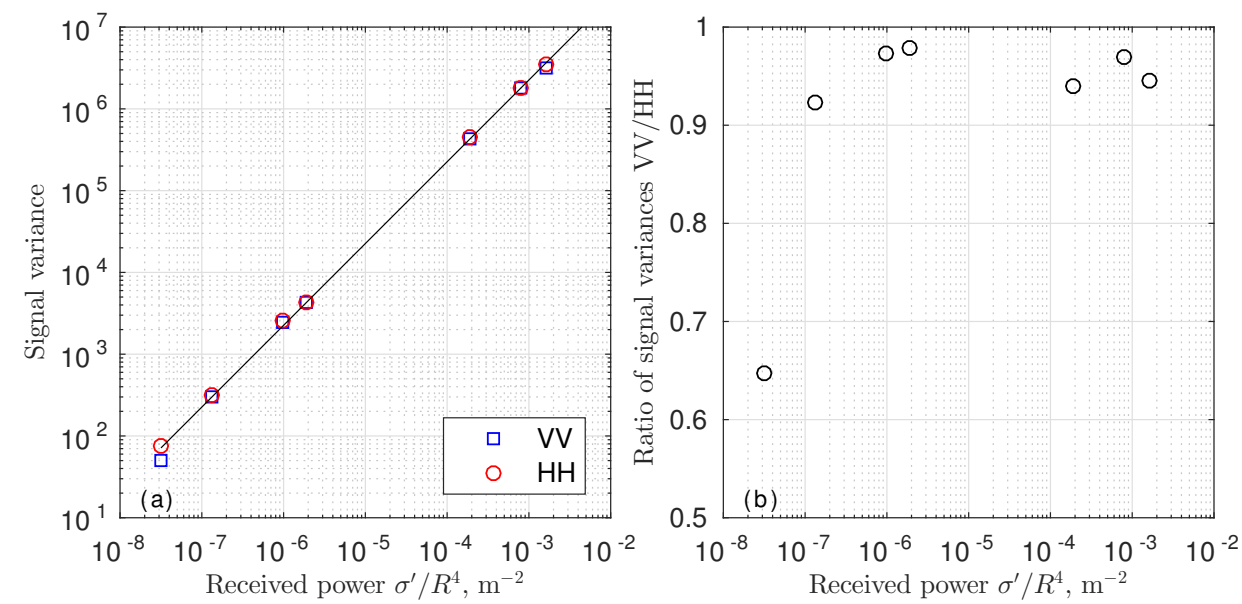

Fig. 19. Dependence (a) of the radar signal variance and (b) ratio of VV and HH variances versus target RCS normalized by the fourth power of distance to the target. The solid line corresponds to the linear fit (calibration curve).

\section{APPENDIX B}

\section{CRoss-Polarized CONTAMINATION}

The radar hybrid polarization mode results in simultaneous receiving of co-polarized (PP) and cross-polarized (CP) signals in a proportion determined by the radar antenna properties. The complex amplitudes of the vertical, $V_{\mathrm{R}}$, and horizontal, $H_{\mathrm{R}}$, received signals are

$$
\left[\begin{array}{l}
V_{\mathrm{R}} \\
H_{\mathrm{R}}
\end{array}\right]=\left[\begin{array}{ll}
R_{\mathrm{vv}} & R_{\mathrm{hv}} \\
R_{\mathrm{vh}} & R_{\mathrm{hh}}
\end{array}\right]\left[\begin{array}{ll}
S_{\mathrm{vv}} & S_{\mathrm{hv}} \\
S_{\mathrm{vh}} & S_{\mathrm{hh}}
\end{array}\right]\left[\begin{array}{ll}
T_{\mathrm{vv}} & T_{\mathrm{hv}} \\
T_{\mathrm{vh}} & T_{\mathrm{hh}}
\end{array}\right]\left[\begin{array}{c}
V_{\mathrm{T}} \\
H_{\mathrm{T}}
\end{array}\right]
$$

where $\mathbf{R}$ and $\mathbf{T}$ are the matrices that describe distortions induced by receive and transmit antennae, respectively, $\mathbf{S}$ is the scattering matrix of the target, $V_{\mathrm{T}}$ and $H_{\mathrm{T}}$ are the intended transmit polarization components, which are equal for our slant polarization mode, $V_{\mathrm{T}}=H_{\mathrm{T}}=1$.

Assuming reasonable polarization isolation (about $-20 \mathrm{~dB}$ for typical horn antenna), $T_{\mathrm{vh}}=T_{\mathrm{hv}}=R_{\mathrm{vh}}=R_{\mathrm{hv}}=$ 0 , and backscattering reciprocal property, $S_{\mathrm{vh}}=S_{\mathrm{hv}}$, equation (26) simplifies to

$$
\left[\begin{array}{c}
V_{\mathrm{R}} \\
H_{\mathrm{R}}
\end{array}\right]=\left[\begin{array}{c}
S_{\mathrm{vv}} T_{\mathrm{vv}} R_{\mathrm{vv}}+S_{\mathrm{vh}} T_{\mathrm{hh}} R_{\mathrm{vv}} \\
S_{\mathrm{hh}} T_{\mathrm{hh}} R_{\mathrm{hh}}+S_{\mathrm{vh}} T_{\mathrm{vv}} R_{\mathrm{hh}}
\end{array}\right] .
$$

From the calibration by non-depolarizing targets (isotropic spheres and trihedral corner reflectors) with known scattering coefficients $S^{\prime}=S_{\mathrm{vv}}^{\prime}=S_{\mathrm{hh}}^{\prime}=\sqrt{\sigma^{\prime}}$, and $S_{\mathrm{vh}}^{\prime}=S_{\mathrm{hv}}^{\prime}=0$, some of the polarization distortion parameters can be found:

$$
\left[\begin{array}{c}
T_{\mathrm{vv}} R_{\mathrm{vv}} \\
T_{\mathrm{hh}} R_{\mathrm{hh}}
\end{array}\right]=\left[\begin{array}{c}
V_{\mathrm{R}}^{\prime} / S^{\prime} \\
H_{\mathrm{R}}^{\prime} / S^{\prime}
\end{array}\right]
$$

The calibration constant for each polarization, $C_{\mathrm{pp}}$, (1-3) can also be determined, $C_{\mathrm{pp}}=T_{\mathrm{pp}}^{2} R_{\mathrm{pp}}^{2} \cdot\left(R^{\prime 4} / \Gamma^{\prime}\right)$. 
The intended calibrated measurements are

$$
\left[\begin{array}{c}
\widetilde{S}_{\mathrm{vv}} \\
\widetilde{S}_{\mathrm{hh}}
\end{array}\right]=\left[\begin{array}{c}
V_{\mathrm{R}} / T_{\mathrm{vv}} R_{\mathrm{vv}} \\
H_{\mathrm{R}} / T_{\mathrm{hh}} R_{\mathrm{hh}}
\end{array}\right] .
$$

For a target with PP return only the measured scattering coefficients are equal to the PP coefficients, $\widetilde{S}_{\mathrm{pp}}=S_{\mathrm{pp}}$. However, for a target producing the CP, equations (27) and (29) suggest that CP signal mixes into the PP signal with coefficients depending on the antenna distortion parameter ratio,

$$
\left[\begin{array}{c}
\widetilde{S}_{\mathrm{vv}} \\
\widetilde{S}_{\mathrm{hh}}
\end{array}\right]=\left[\begin{array}{c}
S_{\mathrm{vv}}+S_{\mathrm{vh}} T_{\mathrm{hh}} / T_{\mathrm{vv}} \\
S_{\mathrm{hh}}+S_{\mathrm{vh}} T_{\mathrm{vv}} / T_{\mathrm{hh}}
\end{array}\right] .
$$

Assuming that $\mathrm{CP}$ and PP are not correlated, $\operatorname{Re}\left(S_{\mathrm{pp}} S_{\mathrm{vh}}^{*}\right)=0$ (see e.g. [43], [44]), the measured scattering cross-sections, $\sigma^{\mathrm{pp}}=\left|S_{\mathrm{pp}}\right|^{2}$, are

$$
\left[\begin{array}{c}
\widetilde{\sigma}^{\mathrm{vv}} \\
\widetilde{\sigma}^{\mathrm{hh}}
\end{array}\right]=\left[\begin{array}{l}
\sigma^{\mathrm{vv}}+\sigma^{\mathrm{vh}} T_{\mathrm{hh}}^{2} / T_{\mathrm{vv}}^{2} \\
\sigma^{\mathrm{hh}}+\sigma^{\mathrm{vh}} T_{\mathrm{vv}}^{2} / T_{\mathrm{hh}}^{2}
\end{array}\right] .
$$

Notice, that rotation of the radar relative to the horizontal plane may also impact the polarized measurements. If the radar is rotated clockwise by an angle, $\gamma$, versus the local horizontal, the scattering matrix in (26) is replaced by

$$
\hat{\mathbf{S}}=\left[\begin{array}{cc}
\cos \gamma & \sin \gamma \\
-\sin \gamma & \cos \gamma
\end{array}\right]\left[\begin{array}{ll}
S_{\mathrm{vv}} & S_{\mathrm{vh}} \\
S_{\mathrm{vh}} & S_{\mathrm{hh}}
\end{array}\right]\left[\begin{array}{cc}
\cos \gamma & -\sin \gamma \\
\sin \gamma & \cos \gamma
\end{array}\right]
$$

However, the radar rotation is controlled with at least $\gamma \approx 1^{\circ}$ accuracy (actually better than that). Simple linear algebra shows that distortions due to the rotation are proportional to at least $\sin ^{2} \gamma \approx-35 \mathrm{~dB}$. This is less than typical polarization isolation for horns $(-20 \mathrm{~dB})$, and thus is not considered.

$\mathrm{CP}$ contribution in (31) leads to the following relative systematic errors, $\delta(x)=(\widetilde{x}-x) / x$, for PP NRCS, $\sigma_{\circ}^{\mathrm{pp}}$, polarization difference (PD), $\Delta \sigma=\sigma_{\circ}^{\mathrm{vv}}-\sigma_{\circ}^{\mathrm{hh}}$, polarization ratio (PR), $P=\sigma_{\circ}^{\mathrm{vv}} / \sigma_{\circ}^{\mathrm{hh}}$, and non-polarized (NP) term:

$$
\begin{aligned}
\delta\left(\sigma_{\circ}^{\mathrm{vv}}\right) & =\frac{\sigma_{\circ}^{\mathrm{vh}}}{\sigma_{\circ}^{\mathrm{vv}}} \cdot \frac{T_{\mathrm{hh}}^{2}}{T_{\mathrm{vv}}^{2}}, \\
\delta\left(\sigma_{\circ}^{\mathrm{hh}}\right) & =\frac{\sigma_{\circ}^{\mathrm{vh}}}{\sigma_{\circ}^{\mathrm{hh}}} \cdot \frac{T_{\mathrm{vv}}^{2}}{T_{\mathrm{hh}}^{2}} \\
\delta(\Delta \sigma) & =\frac{\sigma_{\circ}^{\mathrm{vh}}}{\Delta \sigma} \cdot \frac{\left|T_{\mathrm{hh}}^{4}-T_{\mathrm{vv}}^{4}\right|}{T_{\mathrm{vv}}^{2} T_{\mathrm{hh}}^{2}}, \\
\delta(P) & =\frac{1+\delta\left(\sigma_{\circ}^{\mathrm{vv}}\right)}{1+\delta\left(\sigma_{\circ}^{\mathrm{hh}}\right)}-1 \approx \delta\left(\sigma_{\circ}^{\mathrm{vv}}\right)-\delta\left(\sigma_{\circ}^{\mathrm{hh}}\right), \\
\delta\left(\sigma_{\mathrm{np}}\right) & =\frac{\delta\left(\sigma_{\circ}^{\mathrm{hh}}\right) P_{\mathrm{br}}-\delta\left(\sigma_{\circ}^{\mathrm{vv}}\right) P}{P_{\mathrm{br}}-P} .
\end{aligned}
$$

The first, "scattering" factor in the r.h.s of (33-35) is the ratio between CP and PP (or their combination) and depends on the surface properties only. The second, "antenna" factor is determined by the sensor properties and depends on the polarization isolation of transmitting antenna and how well its polarization plane is aligned with the $45^{\circ}$ plane. The antenna distortion parameters, $T_{\mathrm{pp}}$, are not calibrated separately, but observations in Fig. $19 \mathrm{~b}$ suggest that $\left(T_{\mathrm{vv}}^{2} R_{\mathrm{vv}}^{2}\right) /\left(T_{\mathrm{hh}}^{2} R_{\mathrm{hh}}^{2}\right) \approx 0.9$ or even closer to 1 . Assuming that identical receiving channels have equal 
distortions, $R_{\mathrm{vv}} \approx R_{\mathrm{hh}}$, the transmit antenna distortions can be estimated as $T_{\mathrm{vv}}^{2} / T_{\mathrm{hh}}^{2} \approx 0.9$, and thus the antenna factor for the $\mathrm{PD}$ is $\approx 0.22$.

To evaluate the systematic errors in (33-37), we set all the antenna factors equal to 1, suggesting that the CP contaminates hybrid $\mathrm{PP}$ and $\mathrm{PD}$, so that $\mathrm{PP}=\mathrm{PP}+\mathrm{CP}$ and $\mathrm{PD}=\mathrm{PD}+\mathrm{CP}$. Noting that, $T_{\mathrm{vv}}^{2} / T_{\mathrm{hh}}^{2} \approx 0.9$, (indirectly confirmed by data in Fig. 19b), this will give the upper limit of $\delta(\Delta \sigma)$, and reasonable estimates of $\delta$ for all other variables.

To the best of our knowledge, the CP empirical models are only available for C-band (e.g. [25], [45]). Therefore, in the absence of a better alternative, we assess the errors (33-37) using both C-band CP GMF's and the Two-Scale Model (TSM) predictions. In accordance with the TSM, the ratio CP/PP, reads [34], [41]

$$
\frac{\sigma_{\circ}^{\mathrm{vh}}}{\sigma_{\mathrm{o}}^{\mathrm{pp}}}=\frac{\left|G_{\mathrm{vv}}-G_{\mathrm{hh}}\right|^{2}}{\left|G_{\mathrm{pp}}\right|^{2}} \frac{\overline{\zeta_{\mathrm{c}}^{2}}}{\sin ^{2} \theta}
$$

where $G_{\mathrm{pp}}$ are the Bragg scattering coefficients (see e.g. [34]), $\overline{\zeta_{\mathrm{c}}^{2}}$ is the mean-squared slope of tilting wave in the plane normal to the incidence plane.

Calculations of the relative systematic errors, $\delta$, are shown in Fig. 20 as omni-directional (azimuth-averaged) values versus incidence angle and wind speed. By definition, the TSM does not contain NP scattering, therefore no NP errors for the TSM are shown in Fig. 20.

The estimates based on the empirical data use a combination of co-polarized C-band GMF [24] with different empirical C-band CP models from: i) [25] (see their Table 2a), ii) [45] (see their Table II), iii) [24] (the estimate based on their Fig. 4). Note, that substitution of HH model [24] by that from [45] (see their Table III) does not change the results significantly indicating that HH models from [24], [45] are close.

In general, the TSM-based errors are below the empirical errors. Independent of particular data source, all empirical estimates of CP contamination of measured PP and their derivatives (PR, PD, NP) are small. Note also that Ku-band airborne simultaneous PP and CP measurements [46] (see their Fig. 7) at $U=11 \mathrm{~m} / \mathrm{s}$ and $\theta=44^{\circ}$ suggest the similarly low magnitude of $\delta$ comparable to that in the C-band. This suggests only a minor dependence on radar frequency that allows using empirical C-band estimates as a proxy for our Ka-band.

The estimates shown in the Fig.20 suggest that HH channel and NP term are the most affected by CP contamination, for which up to $20 \%$ error is possible at large incidence angles. We tolerate such error and ignore CP contribution to the hybrid measurements.

\section{APPENDiX C}

\section{EVALUATION OF (4)}

At each iteration step, the equation (4) is evaluated numerically for given $\theta_{0}, \phi_{0}, U$ using the radar antenna pattern (Fig. 18). To improve computational performance the integration in (4) is performed in $\{\alpha, \beta\}$-coordinates (Fig. 21): 

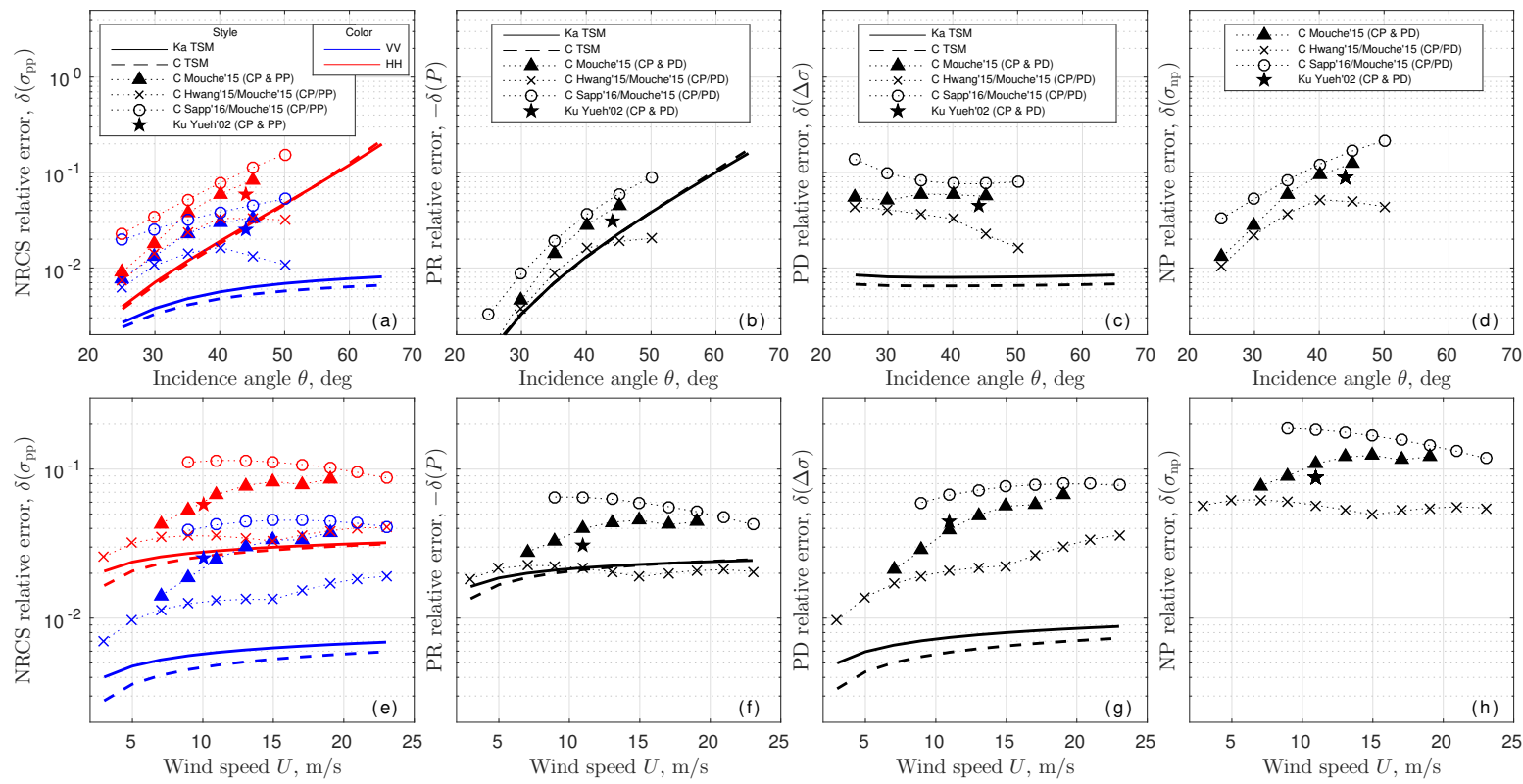

Fig. 20. The relative systematic errors due to the cross-polarized (CP) component for the hybrid omnidirectional (a,e) NRCS (PP+CP), (b,f) polarization ratio $(\mathrm{VV}+\mathrm{CP}) /(\mathrm{HH}+\mathrm{CP}),(\mathrm{c}, \mathrm{g})$ polarization difference $(\mathrm{PD}+\mathrm{CP})$, and $(\mathrm{d}, \mathrm{h})$ non-polarized $(\mathrm{NP})$ term versus $(\mathrm{a}-\mathrm{d})$ incidence angle $\theta$ at $U=15 \mathrm{~m} / \mathrm{s}$ and (e-h) wind speed $U$ at $\theta=45^{\circ}$ from C- and Ka-band TSM prediction, C-band CP GMFs [24], [25], [45] and C-band PP GMF [24]. Ku-band measurements at $U=11 \mathrm{~m} / \mathrm{s}$ and $\theta=44^{\circ}$ [46] are also shown.

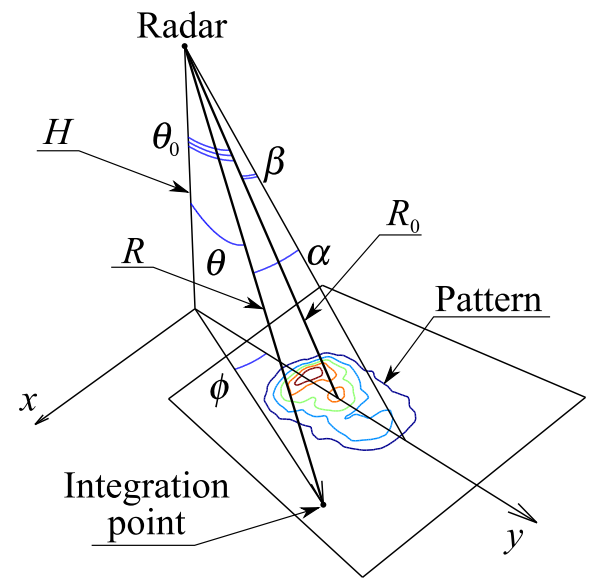

Fig. 21. Radar-look geometry. Sea surface projection of VV pattern faded with slant range is shown. Wind is blowing from $y$-axis direction $\left(\phi_{0}=0\right)$. 


$$
\begin{aligned}
\sigma_{\text {offf }}\left(\theta_{0}, \phi_{0}, U\right) & =\frac{\int \Gamma_{\text {eff }} \sigma_{\circ}(\theta, \phi, U) J \mathrm{~d} \alpha \mathrm{d} \beta}{\int \Gamma_{\text {eff }} J \mathrm{~d} \alpha \mathrm{d} \beta}, \\
\Gamma_{\text {eff }}(\alpha, \beta) & =\Gamma(\alpha, \beta)\left[\frac{R_{0}}{R}\right]^{4}=\Gamma(\alpha, \beta)\left[\frac{\cos \theta}{\cos \theta_{0}}\right]^{4}, \\
\theta(\alpha, \beta) & =\arctan \left\{\left[\frac{\tan ^{2} \alpha}{\cos ^{2} \chi}+\tan ^{2} \chi\right]^{1 / 2}\right\} \\
\phi(\alpha, \beta) & =\phi_{0}+\arctan \left\{\frac{\tan \alpha}{\cos \chi \tan \chi}\right\} \\
J(\alpha, \beta) & =\frac{H^{2}}{\cos ^{3} \chi \cos ^{2} \alpha},
\end{aligned}
$$

where $\chi=\theta_{0}+\beta, H$ is the radar height above the sea surface, and an empirical model for $\sigma_{\circ}(\theta, \phi, U)$ is determined by (6).

\section{APPENDIX D}

$$
\text { Coefficients } C_{m n k} \text { AND } A_{j}(\theta, U)
$$

Coefficients $C_{m n k}$ in (7) are given in Table I ( $\theta$ and $\phi$ are in radians). Coefficients $A_{j}(\theta, U)$ in (7) are tabulated in Tables II and III. They are determined using a method given in [27]:

$$
\begin{aligned}
& A_{0}=\left(\sigma_{\circ}^{\text {up }}+2 \sigma_{\circ}^{\text {cross }}+\sigma_{\circ}^{\text {down }}\right) / 4, \\
& A_{1}=\left(\sigma_{\circ}^{\text {up }}-\sigma_{\circ}^{\text {down }}\right) / 2, \\
& A_{2}=\left(\sigma_{\circ}^{\text {up }}-2 \sigma_{\circ}^{\text {cross }}+\sigma_{\circ}^{\text {down }}\right) / 4 .
\end{aligned}
$$

\section{ACKNOWLEDGMENT}

The authors would like to thank the anonymous referees who made very helpful comments and suggestions leading to improvements of this paper. The work was supported by Russian Science Foundation grant 15-17-20020, and by NASA/Physical Oceanography grant NNX15AG40G.

\section{REFERENCES}

[1] P. Vincent, N. Steunou, E. Caubet, L. Phalippou, L. Rey, E. Thouvenot, and J. Verron, "AltiKa: a Ka-band Altimetry Payload and System for Operational Altimetry during the GMES Period," Sensors, vol. 6, no. 3, pp. 208-234, 2006.

[2] M. Durand, Lee-Lueng Fu, D. P. Lettenmaier, D. E. Alsdorf, E. Rodriguez, and D. Esteban-Fernandez, "The Surface Water and Ocean Topography Mission: Observing Terrestrial Surface Water and Oceanic Submesoscale Eddies," Proc. IEEE, vol. 98, no. 5, pp. 766-779, 2010.

[3] A. Y. Hou, R. K. Kakar, S. Neeck, A. A. Azarbarzin, C. D. Kummerow, M. Kojima, R. Oki, K. Nakamura, and T. Iguchi, "The Global Precipitation Measurement Mission," Bull. Am. Meteor. Soc., vol. 95, pp. 701-722, 2014.

[4] M. Ludwig, E. Daganzo-Eusebio, and M. Davidson, "Ka-Band radar missions for earth observation," in Proc. Int. Geosci. Rem. Sens. Symp. IGARSS'13, Melbourne, VIC, Jul. 2013, pp. 2289-2292. 
TABLE I

COEFFICIENTS $C_{m n k}$

\begin{tabular}{|c|c|c|c|c|}
\hline \multicolumn{3}{|c|}{ Index } & \multicolumn{2}{|c|}{$C_{m n k}$} \\
\hline$m$ & $n$ & $k$ & VV & $\mathrm{HH}$ \\
\hline 0 & 0 & 0 & $+3.206118 \cdot 10^{+0}$ & $+3.287958 \cdot 10^{+0}$ \\
\hline 1 & 0 & 0 & $+1.951546 \cdot 10^{+0}$ & $+2.958732 \cdot 10^{-2}$ \\
\hline 2 & 0 & 0 & $-7.208258 \cdot 10^{+1}$ & $-6.570137 \cdot 10^{+1}$ \\
\hline 3 & 0 & 0 & $+8.578391 \cdot 10^{+1}$ & $+7.779126 \cdot 10^{+1}$ \\
\hline 4 & 0 & 0 & $-2.884517 \cdot 10^{+1}$ & $-2.641669 \cdot 10^{+1}$ \\
\hline 0 & 1 & 0 & $-3.791021 \cdot 10^{-2}$ & $-6.110719 \cdot 10^{-2}$ \\
\hline 1 & 1 & 0 & $+4.193799 \cdot 10^{+0}$ & $+3.088378 \cdot 10^{+0}$ \\
\hline 2 & 1 & 0 & $-1.337898 \cdot 10^{+1}$ & $-1.109291 \cdot 10^{+1}$ \\
\hline 3 & 1 & 0 & $+1.119162 \cdot 10^{+1}$ & $+1.105847 \cdot 10^{+1}$ \\
\hline 4 & 1 & 0 & $-2.305322 \cdot 10^{+0}$ & $-2.403804 \cdot 10^{+0}$ \\
\hline 0 & 2 & 0 & $+1.123723 \cdot 10^{-2}$ & $+3.093813 \cdot 10^{-2}$ \\
\hline 1 & 2 & 0 & $+7.798137 \cdot 10^{+0}$ & $+6.490559 \cdot 10^{+0}$ \\
\hline 2 & 2 & 0 & $-3.132253 \cdot 10^{+1}$ & $-3.154284 \cdot 10^{+1}$ \\
\hline 3 & 2 & 0 & $+4.686008 \cdot 10^{+1}$ & $+4.898348 \cdot 10^{+1}$ \\
\hline 4 & 2 & 0 & $-2.244278 \cdot 10^{+1}$ & $-2.351261 \cdot 10^{+1}$ \\
\hline 0 & 0 & 1 & $-2.007813 \cdot 10^{-1}$ & $-1.435727 \cdot 10^{-1}$ \\
\hline 1 & 0 & 1 & $-1.556322 \cdot 10^{+0}$ & $-1.614046 \cdot 10^{+0}$ \\
\hline 2 & 0 & 1 & $+1.779589 \cdot 10^{+1}$ & $+1.771247 \cdot 10^{+1}$ \\
\hline 3 & 0 & 1 & $-1.905703 \cdot 10^{+1}$ & $-2.040338 \cdot 10^{+1}$ \\
\hline 4 & 0 & 1 & $+5.425915 \cdot 10^{+0}$ & $+6.773906 \cdot 10^{+0}$ \\
\hline 0 & 1 & 1 & $+2.754555 \cdot 10^{-2}$ & $+2.209574 \cdot 10^{-2}$ \\
\hline 1 & 1 & 1 & $-2.375674 \cdot 10^{+0}$ & $-1.987757 \cdot 10^{+0}$ \\
\hline 2 & 1 & 1 & $+7.034096 \cdot 10^{+0}$ & $+6.865252 \cdot 10^{+0}$ \\
\hline 3 & 1 & 1 & $-5.337939 \cdot 10^{+0}$ & $-6.369661 \cdot 10^{+0}$ \\
\hline 4 & 1 & 1 & $+9.388563 \cdot 10^{-1}$ & $+1.467463 \cdot 10^{+0}$ \\
\hline 0 & 2 & 1 & $-4.769737 \cdot 10^{-3}$ & $-4.955172 \cdot 10^{-3}$ \\
\hline 1 & 2 & 1 & $-4.252548 \cdot 10^{+0}$ & $-3.603769 \cdot 10^{+0}$ \\
\hline 2 & 2 & 1 & $+1.943467 \cdot 10^{+1}$ & $+1.922202 \cdot 10^{+1}$ \\
\hline 3 & 2 & 1 & $-2.873040 \cdot 10^{+1}$ & $-2.904522 \cdot 10^{+1}$ \\
\hline 4 & 2 & 1 & $+1.330676 \cdot 10^{+1}$ & $+1.332051 \cdot 10^{+1}$ \\
\hline
\end{tabular}

[5] J. F. Nouvel, P. Dubois-Fernandez, and X. Dupuis, "The KaSAR airborne campaign," in Proc. Int. Geosci. Rem. Sens. Symp. IGARSS'13, Melbourne, VIC, Jul. 2013, pp. 4471-4474.

[6] D. Mapelli, N. Pierdicca, L. Guerriero, P. Ferrazzoli, E. Calleja, B. Rommen, D. Giudici, and A. Monti Guarnieri, "A comparative study of RADAR Ka-band backscatter," in Proc. SPIE SAR Image Analysis, Modeling, and Techniques XIV, vol. 9243, Oct. 2014.

[7] A. W. Doerry, D. F. Dubbert, M. Thompson, and V. D. Gutierrez, "A portfolio of fine resolution Ka-band SAR images: part I," in Proc. SPIE Defense and Security Symposium, vol. 5788, May 2005, pp. 13-24. [Online]. Available: http://www.sandia.gov/radar/Web/images/SAND-2005-1704P-Oral-pres-slide-show.pdf

[8] B. Chapron, F. Collard, and F. Ardhuin, "Direct measurements of ocean surface velocity from space: Interpretation and validation," $J$. Geophys. Res. Oceans, vol. 110, no. C7, 2005, doi: 10.1029/2004JC002809.

[9] J. A. Johannessen, B. Chapron, F. Collard, V. Kudryavtsev, A. Mouche, D. Akimov, and K.-F. Dagestad, "Direct ocean surface velocity measurements from space: Improved quantitative interpretation of envisat asar observations,” Geophys. Res. Lett., vol. 35, no. 22, 2008, doi: 10.1029/2008GL035709.

[10] E. Rodriguez, D. Perkovic-Martin, C. Baldi, K. Cooper, N. Majurec, M. Neumann, F. Nicaise, and G. Farquharson, "Ka-band Doppler Scatterometer for Measurements of Ocean Surface Vector Winds and Currents," in Proc. Earth Sci. Tech. Forum ESTF2014, Leesburg, Virginia, USA, Oct. 2014.

[11] H. Hersbach, "Comparison of C-Band Scatterometer CMOD5.N Equivalent Neutral Winds with ECMWF," J. Atmos. Ocean. Tech., vol. 27, pp. 721-736, Apr. 2010.

[12] QuikSCAT Science Data Product User's Manual Overview and Geophysical Data Products, Jet Propulsion Laboratory, 2006. [Online]. Available: ftp://podaac.jpl.nasa.gov/allData/quikscat/L2A/v2/docs/QSUG_v3.pdf

[13] F. J. Wentz and D. K. Smith, "A model function for the ocean-normalized radar cross section at $14 \mathrm{GHz}$ derived from NSCAT observations," J. Geophys. Res. Oceans, vol. 104, no. C5, pp. $11499-11514,1999$. 
TABLE II

COEFFICIENTS $A_{j}(\theta, U)$ FOR VV POLARIZATION

\begin{tabular}{|c|c|c|c|c|c|c|c|c|}
\hline \multirow{2}{*}{$\theta,{ }^{\circ}$} & \multicolumn{8}{|c|}{ Wind speed $U, \mathrm{~m} / \mathrm{s}$} \\
\hline & 3 & 5 & 7 & 9 & 11 & 13 & 15 & 17 \\
\hline \multicolumn{9}{|c|}{$A_{0}$} \\
\hline 25 & $+1.05 \cdot 10^{-1}$ & $+1.84 \cdot 10^{-1}$ & $+2.67 \cdot 10^{-1}$ & $+3.52 \cdot 10^{-1}$ & $+4.39 \cdot 10^{-1}$ & $+5.28 \cdot 10^{-1}$ & $+6.18 \cdot 10^{-1}$ & $+7.10 \cdot 10^{-1}$ \\
\hline 30 & $+2.81 \cdot 10^{-2}$ & $+6.12 \cdot 10^{-2}$ & $+1.02 \cdot 10^{-1}$ & $+1.50 \cdot 10^{-1}$ & $+2.03 \cdot 10^{-1}$ & $+2.62 \cdot 10^{-1}$ & $+3.26 \cdot 10^{-1}$ & $+3.94 \cdot 10^{-1}$ \\
\hline 35 & $+9.07 \cdot 10^{-3}$ & $+2.36 \cdot 10^{-2}$ & $+4.43 \cdot 10^{-2}$ & $+7.09 \cdot 10^{-2}$ & $+1.03 \cdot 10^{-1}$ & $+1.42 \cdot 10^{-1}$ & $+1.86 \cdot 10^{-1}$ & $+2.35 \cdot 10^{-1}$ \\
\hline 40 & $+3.70 \cdot 10^{-3}$ & $+1.09 \cdot 10^{-2}$ & $+2.22 \cdot 10^{-2}$ & $+3.81 \cdot 10^{-2}$ & $+5.86 \cdot 10^{-2}$ & $+8.40 \cdot 10^{-2}$ & $+1.14 \cdot 10^{-1}$ & $+1.50 \cdot 10^{-1}$ \\
\hline 45 & $+1.92 \cdot 10^{-3}$ & $+6.03 \cdot 10^{-3}$ & $+1.30 \cdot 10^{-2}$ & $+2.32 \cdot 10^{-2}$ & $+3.69 \cdot 10^{-2}$ & $+5.46 \cdot 10^{-2}$ & $+7.64 \cdot 10^{-2}$ & $+1.03 \cdot 10^{-1}$ \\
\hline 50 & $+1.22 \cdot 10^{-3}$ & $+3.93 \cdot 10^{-3}$ & $+8.65 \cdot 10^{-3}$ & $+1.57 \cdot 10^{-2}$ & $+2.55 \cdot 10^{-2}$ & $+3.83 \cdot 10^{-2}$ & $+5.43 \cdot 10^{-2}$ & $+7.39 \cdot 10^{-2}$ \\
\hline 55 & $+8.88 \cdot 10^{-4}$ & $+2.87 \cdot 10^{-3}$ & $+6.31 \cdot 10^{-3}$ & $+1.15 \cdot 10^{-2}$ & $+1.86 \cdot 10^{-2}$ & $+2.80 \cdot 10^{-2}$ & $+3.97 \cdot 10^{-2}$ & $+5.39 \cdot 10^{-2}$ \\
\hline 60 & $+6.77 \cdot 10^{-4}$ & $+2.18 \cdot 10^{-3}$ & $+4.73 \cdot 10^{-3}$ & $+8.48 \cdot 10^{-3}$ & $+1.35 \cdot 10^{-2}$ & $+2.00 \cdot 10^{-2}$ & $+2.80 \cdot 10^{-2}$ & $+3.76 \cdot 10^{-2}$ \\
\hline 65 & $+5.21 \cdot 10^{-4}$ & $+1.58 \cdot 10^{-3}$ & $+3.32 \cdot 10^{-3}$ & $+5.81 \cdot 10^{-3}$ & $+9.14 \cdot 10^{-3}$ & $+1.34 \cdot 10^{-2}$ & $+1.85 \cdot 10^{-2}$ & $+2.47 \cdot 10^{-2}$ \\
\hline \multicolumn{9}{|c|}{$A_{1}$} \\
\hline 25 & $+5.86 \cdot 10^{-4}$ & $-9.45 \cdot 10^{-3}$ & $-2.33 \cdot 10^{-2}$ & $-4.00 \cdot 10^{-2}$ & $-5.90 \cdot 10^{-2}$ & $-7.98 \cdot 10^{-2}$ & $-1.02 \cdot 10^{-1}$ & $-1.26 \cdot 10^{-1}$ \\
\hline 30 & $-2.43 \cdot 10^{-3}$ & $-4.50 \cdot 10^{-3}$ & $-6.66 \cdot 10^{-3}$ & $-8.83 \cdot 10^{-3}$ & $-1.10 \cdot 10^{-2}$ & $-1.31 \cdot 10^{-2}$ & $-1.52 \cdot 10^{-2}$ & $-1.71 \cdot 10^{-2}$ \\
\hline 35 & $-1.55 \cdot 10^{-3}$ & $-1.89 \cdot 10^{-3}$ & $-9.60 \cdot 10^{-4}$ & $+1.54 \cdot 10^{-3}$ & $+5.78 \cdot 10^{-3}$ & $+1.19 \cdot 10^{-2}$ & $+2.00 \cdot 10^{-2}$ & $+3.03 \cdot 10^{-2}$ \\
\hline 40 & $-8.55 \cdot 10^{-4}$ & $-7.06 \cdot 10^{-4}$ & $+9.03 \cdot 10^{-4}$ & $+4.45 \cdot 10^{-3}$ & $+1.03 \cdot 10^{-2}$ & $+1.88 \cdot 10^{-2}$ & $+3.01 \cdot 10^{-2}$ & $+4.46 \cdot 10^{-2}$ \\
\hline 45 & $-4.78 \cdot 10^{-4}$ & $-1.37 \cdot 10^{-4}$ & $+1.51 \cdot 10^{-3}$ & $+4.93 \cdot 10^{-3}$ & $+1.05 \cdot 10^{-2}$ & $+1.86 \cdot 10^{-2}$ & $+2.96 \cdot 10^{-2}$ & $+4.36 \cdot 10^{-2}$ \\
\hline 50 & $-2.56 \cdot 10^{-4}$ & $+1.82 \cdot 10^{-4}$ & $+1.70 \cdot 10^{-3}$ & $+4.68 \cdot 10^{-3}$ & $+9.42 \cdot 10^{-3}$ & $+1.62 \cdot 10^{-2}$ & $+2.53 \cdot 10^{-2}$ & $+3.69 \cdot 10^{-2}$ \\
\hline 55 & $-9.15 \cdot 10^{-5}$ & $+3.93 \cdot 10^{-4}$ & $+1.73 \cdot 10^{-3}$ & $+4.19 \cdot 10^{-3}$ & $+7.97 \cdot 10^{-3}$ & $+1.33 \cdot 10^{-2}$ & $+2.03 \cdot 10^{-2}$ & $+2.91 \cdot 10^{-2}$ \\
\hline 60 & $+3.92 \cdot 10^{-5}$ & $+5.13 \cdot 10^{-4}$ & $+1.62 \cdot 10^{-3}$ & $+3.54 \cdot 10^{-3}$ & $+6.40 \cdot 10^{-3}$ & $+1.03 \cdot 10^{-2}$ & $+1.55 \cdot 10^{-2}$ & $+2.19 \cdot 10^{-2}$ \\
\hline 65 & $+1.05 \cdot 10^{-4}$ & $+4.98 \cdot 10^{-4}$ & $+1.33 \cdot 10^{-3}$ & $+2.71 \cdot 10^{-3}$ & $+4.75 \cdot 10^{-3}$ & $+7.56 \cdot 10^{-3}$ & $+1.12 \cdot 10^{-2}$ & $+1.58 \cdot 10^{-2}$ \\
\hline \multicolumn{9}{|c|}{$A_{2}$} \\
\hline 25 & $+4.52 \cdot 10^{-2}$ & $+7.41 \cdot 10^{-2}$ & $+1.03 \cdot 10^{-1}$ & $+1.31 \cdot 10^{-1}$ & $+1.58 \cdot 10^{-1}$ & $+1.86 \cdot 10^{-1}$ & $+2.13 \cdot 10^{-1}$ & $+2.40 \cdot 10^{-1}$ \\
\hline 30 & $+1.34 \cdot 10^{-2}$ & $+2.84 \cdot 10^{-2}$ & $+4.67 \cdot 10^{-2}$ & $+6.77 \cdot 10^{-2}$ & $+9.09 \cdot 10^{-2}$ & $+1.16 \cdot 10^{-1}$ & $+1.44 \cdot 10^{-1}$ & $+1.73 \cdot 10^{-1}$ \\
\hline 35 & $+4.84 \cdot 10^{-3}$ & $+1.21 \cdot 10^{-2}$ & $+2.22 \cdot 10^{-2}$ & $+3.50 \cdot 10^{-2}$ & $+5.04 \cdot 10^{-2}$ & $+6.82 \cdot 10^{-2}$ & $+8.84 \cdot 10^{-2}$ & $+1.11 \cdot 10^{-1}$ \\
\hline 40 & $+2.19 \cdot 10^{-3}$ & $+5.96 \cdot 10^{-3}$ & $+1.16 \cdot 10^{-2}$ & $+1.90 \cdot 10^{-2}$ & $+2.83 \cdot 10^{-2}$ & $+3.95 \cdot 10^{-2}$ & $+5.25 \cdot 10^{-2}$ & $+6.75 \cdot 10^{-2}$ \\
\hline 45 & $+1.22 \cdot 10^{-3}$ & $+3.39 \cdot 10^{-3}$ & $+6.65 \cdot 10^{-3}$ & $+1.10 \cdot 10^{-2}$ & $+1.64 \cdot 10^{-2}$ & $+2.29 \cdot 10^{-2}$ & $+3.05 \cdot 10^{-2}$ & $+3.91 \cdot 10^{-2}$ \\
\hline 50 & $+7.92 \cdot 10^{-4}$ & $+2.17 \cdot 10^{-3}$ & $+4.17 \cdot 10^{-3}$ & $+6.76 \cdot 10^{-3}$ & $+9.87 \cdot 10^{-3}$ & $+1.34 \cdot 10^{-2}$ & $+1.74 \cdot 10^{-2}$ & $+2.17 \cdot 10^{-2}$ \\
\hline 55 & $+5.41 \cdot 10^{-4}$ & $+1.45 \cdot 10^{-3}$ & $+2.75 \cdot 10^{-3}$ & $+4.38 \cdot 10^{-3}$ & $+6.30 \cdot 10^{-3}$ & $+8.44 \cdot 10^{-3}$ & $+1.08 \cdot 10^{-2}$ & $+1.32 \cdot 10^{-2}$ \\
\hline 60 & $+3.14 \cdot 10^{-4}$ & $+8.99 \cdot 10^{-4}$ & $+1.81 \cdot 10^{-3}$ & $+3.05 \cdot 10^{-3}$ & $+4.65 \cdot 10^{-3}$ & $+6.61 \cdot 10^{-3}$ & $+8.95 \cdot 10^{-3}$ & $+1.17 \cdot 10^{-2}$ \\
\hline 65 & $+5.32 \cdot 10^{-5}$ & $+3.89 \cdot 10^{-4}$ & $+1.12 \cdot 10^{-3}$ & $+2.34 \cdot 10^{-3}$ & $+4.13 \cdot 10^{-3}$ & $+6.56 \cdot 10^{-3}$ & $+9.70 \cdot 10^{-3}$ & $+1.36 \cdot 10^{-2}$ \\
\hline
\end{tabular}

[14] Y. Quilfen, B. Chapron, A. Bentamy, J. Gourrion, T. Elfouhaily, and D. Vandemark, "Global ERS 1 and 2 and NSCAT observations: Upwind/crosswind and upwind/downwind measurements," J. Geophys. Res. Oceans, vol. 104, no. C5, pp. 11459-11469, 1999.

[15] H. Masuko, K. Okamoto, M. Shimada, and S. Niwa, "Measurement of microwave backscattering signatures of the ocean surface using X band and Ka band airborne scatterometers," J. Geophys. Res. Oceans, vol. 91, pp. 13065-13084, 1986.

[16] A. Nekrasov and P. Hoogeboom, "A Ka-Band Backscatter Model Function and an Algorithm for Measurement of the Wind Vector Over the Sea Surface," IEEE Geosci. Remote Sens. Lett., vol. 2, pp. 23-27, 2005.

[17] E. J. Walsh, D. C. Vandemark, C. A. Friehe, S. P. Burns, D. Khelif, R. N. Swift, and J. F. Scott, "Measuring sea surface mean square slope with a 36-GHz scanning radar altimeter," J. Geophys. Res. Oceans, vol. 103, no. C6, pp. 12 587-12 601, Jun. 1998.

[18] D. Vandemark, B. Chapron, J. Sun, G. H. Crescenti, and H. C. Graber, "Ocean Wave Slope Observations Using Radar Backscatter and Laser Altimeters," J. Phys. Oceanogr., vol. 34, pp. 2825-2842, 2004.

[19] S. Tanelli, S. L. Durden, and E. Im, "Simultaneous Measurements of Ku- and Ka-Band Sea Surface Cross Sections by an Airborne Radar," IEEE Geosci. Remote Sens. Lett., vol. 3, pp. 359-363, 2006.

[20] W. J. Plant, E. A. Terray, R. A. Petitt, and W. C. Keller, "The dependence of microwave backscatter from the sea on illuminated area: Correlation times and lengths," J. Geophys. Res. Oceans, vol. 99, pp. 9705-9723, 1994.

[21] J.-P. Giovanangeli, L. F. Bliven, and O. Le Calve, “A wind-wave tank study of the azimuthal response of a Ka-band scatterometer," IEEE Trans. Geosci. Remote Sens., vol. 29, pp. 143-148, Jan. 1991.

[22] O. Boisot, S. Pioch, C. Fatras, G. Caulliez, A. Bringer, P. Borderies, J.-C. Lalaurie, and C.-A. Guérin, "Ka-band backscattering from water surface at small incidence: A wind-wave tank study," J. Geophys. Res. Oceans, vol. 120, pp. 3261-3285, 2015.

[23] G.-J. van Zadelhoff, A. Stoffelen, P. W. Vachon, J. Wolfe, J. Horstmann, and M. Belmonte Rivas, "Retrieving hurricane wind speeds using cross-polarization C-band measurements," Atmospheric Measurement Techniques, vol. 7, no. 2, pp. 437-449, 2014.

[24] A. Mouche and B. Chapron, "Global C-Band Envisat, RADARSAT-2 and Sentinel-1 SAR measurements in copolarization and cross- 
TABLE III

COEFFICIENTS $A_{j}(\theta, U)$ FOR HH POLARIZATION

\begin{tabular}{|c|c|c|c|c|c|c|c|c|}
\hline \multirow{2}{*}{$\theta,{ }^{\circ}$} & \multicolumn{8}{|c|}{ Wind speed $U, \mathrm{~m} / \mathrm{s}$} \\
\hline & 3 & 5 & 7 & 9 & 11 & 13 & 15 & 17 \\
\hline \multicolumn{9}{|c|}{$A_{0}$} \\
\hline 25 & $+8.27 \cdot 10^{-2}$ & $+1.46 \cdot 10^{-1}$ & $+2.14 \cdot 10^{-1}$ & $+2.84 \cdot 10^{-1}$ & $+3.56 \cdot 10^{-1}$ & $+4.31 \cdot 10^{-1}$ & $+5.08 \cdot 10^{-1}$ & $+5.86 \cdot 10^{-1}$ \\
\hline 30 & $+2.02 \cdot 10^{-2}$ & $+4.39 \cdot 10^{-2}$ & $+7.35 \cdot 10^{-2}$ & $+1.08 \cdot 10^{-1}$ & $+1.48 \cdot 10^{-1}$ & $+1.92 \cdot 10^{-1}$ & $+2.40 \cdot 10^{-1}$ & $+2.92 \cdot 10^{-1}$ \\
\hline 35 & $+5.78 \cdot 10^{-3}$ & $+1.49 \cdot 10^{-2}$ & $+2.79 \cdot 10^{-2}$ & $+4.47 \cdot 10^{-2}$ & $+6.54 \cdot 10^{-2}$ & $+8.98 \cdot 10^{-2}$ & $+1.18 \cdot 10^{-1}$ & $+1.50 \cdot 10^{-1}$ \\
\hline 40 & $+2.02 \cdot 10^{-3}$ & $+5.88 \cdot 10^{-3}$ & $+1.20 \cdot 10^{-2}$ & $+2.04 \cdot 10^{-2}$ & $+3.12 \cdot 10^{-2}$ & $+4.46 \cdot 10^{-2}$ & $+6.05 \cdot 10^{-2}$ & $+7.91 \cdot 10^{-2}$ \\
\hline 45 & $+8.78 \cdot 10^{-4}$ & $+2.75 \cdot 10^{-3}$ & $+5.87 \cdot 10^{-3}$ & $+1.03 \cdot 10^{-2}$ & $+1.63 \cdot 10^{-2}$ & $+2.37 \cdot 10^{-2}$ & $+3.28 \cdot 10^{-2}$ & $+4.36 \cdot 10^{-2}$ \\
\hline 50 & $+4.70 \cdot 10^{-4}$ & $+1.52 \cdot 10^{-3}$ & $+3.29 \cdot 10^{-3}$ & $+5.88 \cdot 10^{-3}$ & $+9.34 \cdot 10^{-3}$ & $+1.37 \cdot 10^{-2}$ & $+1.91 \cdot 10^{-2}$ & $+2.56 \cdot 10^{-2}$ \\
\hline 55 & $+2.97 \cdot 10^{-4}$ & $+9.59 \cdot 10^{-4}$ & $+2.08 \cdot 10^{-3}$ & $+3.71 \cdot 10^{-3}$ & $+5.89 \cdot 10^{-3}$ & $+8.67 \cdot 10^{-3}$ & $+1.21 \cdot 10^{-2}$ & $+1.61 \cdot 10^{-2}$ \\
\hline 60 & $+2.10 \cdot 10^{-4}$ & $+6.69 \cdot 10^{-4}$ & $+1.43 \cdot 10^{-3}$ & $+2.54 \cdot 10^{-3}$ & $+4.00 \cdot 10^{-3}$ & $+5.84 \cdot 10^{-3}$ & $+8.09 \cdot 10^{-3}$ & $+1.08 \cdot 10^{-2}$ \\
\hline 65 & $+1.66 \cdot 10^{-4}$ & $+4.96 \cdot 10^{-4}$ & $+1.03 \cdot 10^{-3}$ & $+1.79 \cdot 10^{-3}$ & $+2.79 \cdot 10^{-3}$ & $+4.04 \cdot 10^{-3}$ & $+5.57 \cdot 10^{-3}$ & $+7.37 \cdot 10^{-3}$ \\
\hline \multicolumn{9}{|c|}{$A_{1}$} \\
\hline 25 & $-9.70 \cdot 10^{-4}$ & $-3.20 \cdot 10^{-3}$ & $-6.21 \cdot 10^{-3}$ & $-9.85 \cdot 10^{-3}$ & $-1.40 \cdot 10^{-2}$ & $-1.87 \cdot 10^{-2}$ & $-2.38 \cdot 10^{-2}$ & $-2.92 \cdot 10^{-2}$ \\
\hline 30 & $-3.40 \cdot 10^{-4}$ & $+1.03 \cdot 10^{-3}$ & $+3.89 \cdot 10^{-3}$ & $+8.27 \cdot 10^{-3}$ & $+1.42 \cdot 10^{-2}$ & $+2.16 \cdot 10^{-2}$ & $+3.05 \cdot 10^{-2}$ & $+4.10 \cdot 10^{-2}$ \\
\hline 35 & $+4.75 \cdot 10^{-5}$ & $+1.44 \cdot 10^{-3}$ & $+4.47 \cdot 10^{-3}$ & $+9.41 \cdot 10^{-3}$ & $+1.64 \cdot 10^{-2}$ & $+2.57 \cdot 10^{-2}$ & $+3.74 \cdot 10^{-2}$ & $+5.15 \cdot 10^{-2}$ \\
\hline 40 & $+1.62 \cdot 10^{-4}$ & $+1.19 \cdot 10^{-3}$ & $+3.42 \cdot 10^{-3}$ & $+7.12 \cdot 10^{-3}$ & $+1.25 \cdot 10^{-2}$ & $+1.98 \cdot 10^{-2}$ & $+2.92 \cdot 10^{-2}$ & $+4.08 \cdot 10^{-2}$ \\
\hline 45 & $+1.89 \cdot 10^{-4}$ & $+9.31 \cdot 10^{-4}$ & $+2.45 \cdot 10^{-3}$ & $+4.94 \cdot 10^{-3}$ & $+8.53 \cdot 10^{-3}$ & $+1.34 \cdot 10^{-2}$ & $+1.96 \cdot 10^{-2}$ & $+2.72 \cdot 10^{-2}$ \\
\hline 50 & $+1.94 \cdot 10^{-4}$ & $+7.50 \cdot 10^{-4}$ & $+1.79 \cdot 10^{-3}$ & $+3.40 \cdot 10^{-3}$ & $+5.66 \cdot 10^{-3}$ & $+8.62 \cdot 10^{-3}$ & $+1.24 \cdot 10^{-2}$ & $+1.69 \cdot 10^{-2}$ \\
\hline 55 & $+1.90 \cdot 10^{-4}$ & $+6.20 \cdot 10^{-4}$ & $+1.35 \cdot 10^{-3}$ & $+2.41 \cdot 10^{-3}$ & $+3.82 \cdot 10^{-3}$ & $+5.61 \cdot 10^{-3}$ & $+7.80 \cdot 10^{-3}$ & $+1.04 \cdot 10^{-2}$ \\
\hline 60 & $+1.68 \cdot 10^{-4}$ & $+5.02 \cdot 10^{-4}$ & $+1.03 \cdot 10^{-3}$ & $+1.76 \cdot 10^{-3}$ & $+2.69 \cdot 10^{-3}$ & $+3.83 \cdot 10^{-3}$ & $+5.19 \cdot 10^{-3}$ & $+6.77 \cdot 10^{-3}$ \\
\hline 65 & $+1.20 \cdot 10^{-4}$ & $+3.69 \cdot 10^{-4}$ & $+7.65 \cdot 10^{-4}$ & $+1.31 \cdot 10^{-3}$ & $+2.01 \cdot 10^{-3}$ & $+2.85 \cdot 10^{-3}$ & $+3.83 \cdot 10^{-3}$ & $+4.95 \cdot 10^{-3}$ \\
\hline \multicolumn{9}{|c|}{$A_{2}$} \\
\hline 25 & $+1.96 \cdot 10^{-2}$ & $+4.52 \cdot 10^{-2}$ & $+7.57 \cdot 10^{-2}$ & $+1.10 \cdot 10^{-1}$ & $+1.47 \cdot 10^{-1}$ & $+1.87 \cdot 10^{-1}$ & $+2.29 \cdot 10^{-1}$ & $+2.74 \cdot 10^{-1}$ \\
\hline 30 & $+5.46 \cdot 10^{-3}$ & $+1.61 \cdot 10^{-2}$ & $+3.13 \cdot 10^{-2}$ & $+5.07 \cdot 10^{-2}$ & $+7.40 \cdot 10^{-2}$ & $+1.01 \cdot 10^{-1}$ & $+1.32 \cdot 10^{-1}$ & $+1.65 \cdot 10^{-1}$ \\
\hline 35 & $+1.88 \cdot 10^{-3}$ & $+6.16 \cdot 10^{-3}$ & $+1.31 \cdot 10^{-2}$ & $+2.28 \cdot 10^{-2}$ & $+3.53 \cdot 10^{-2}$ & $+5.07 \cdot 10^{-2}$ & $+6.90 \cdot 10^{-2}$ & $+9.02 \cdot 10^{-2}$ \\
\hline 40 & $+7.89 \cdot 10^{-4}$ & $+2.64 \cdot 10^{-3}$ & $+5.81 \cdot 10^{-3}$ & $+1.05 \cdot 10^{-2}$ & $+1.67 \cdot 10^{-2}$ & $+2.46 \cdot 10^{-2}$ & $+3.44 \cdot 10^{-2}$ & $+4.60 \cdot 10^{-2}$ \\
\hline 45 & $+3.93 \cdot 10^{-4}$ & $+1.27 \cdot 10^{-3}$ & $+2.77 \cdot 10^{-3}$ & $+4.97 \cdot 10^{-3}$ & $+7.94 \cdot 10^{-3}$ & $+1.17 \cdot 10^{-2}$ & $+1.64 \cdot 10^{-2}$ & $+2.20 \cdot 10^{-2}$ \\
\hline 50 & $+2.22 \cdot 10^{-4}$ & $+6.76 \cdot 10^{-4}$ & $+1.41 \cdot 10^{-3}$ & $+2.44 \cdot 10^{-3}$ & $+3.78 \cdot 10^{-3}$ & $+5.45 \cdot 10^{-3}$ & $+7.45 \cdot 10^{-3}$ & $+9.80 \cdot 10^{-3}$ \\
\hline 55 & $+1.30 \cdot 10^{-4}$ & $+3.69 \cdot 10^{-4}$ & $+7.28 \cdot 10^{-4}$ & $+1.20 \cdot 10^{-3}$ & $+1.78 \cdot 10^{-3}$ & $+2.45 \cdot 10^{-3}$ & $+3.23 \cdot 10^{-3}$ & $+4.08 \cdot 10^{-3}$ \\
\hline 60 & $+6.06 \cdot 10^{-5}$ & $+1.74 \cdot 10^{-4}$ & $+3.45 \cdot 10^{-4}$ & $+5.76 \cdot 10^{-4}$ & $+8.63 \cdot 10^{-4}$ & $+1.21 \cdot 10^{-3}$ & $+1.61 \cdot 10^{-3}$ & $+2.06 \cdot 10^{-3}$ \\
\hline 65 & $-1.09 \cdot 10^{-5}$ & $+1.84 \cdot 10^{-5}$ & $+1.10 \cdot 10^{-4}$ & $+2.87 \cdot 10^{-4}$ & $+5.65 \cdot 10^{-4}$ & $+9.63 \cdot 10^{-4}$ & $+1.50 \cdot 10^{-3}$ & $+2.18 \cdot 10^{-3}$ \\
\hline
\end{tabular}

polarization," J. Geophys. Res. Oceans, vol. 120, no. 11, pp. 7195-7207, 2015.

[25] P. A. Hwang, A. Stoffelen, G.-J. van Zadelhoff, W. Perrie, B. Zhang, H. Li, and H. Shen, "Cross-polarization geophysical model function for C-band radar backscattering from the ocean surface and wind speed retrieval," J. Geophys. Res. Oceans, vol. 120, no. 2, pp. 893-909, 2015.

[26] C. W. Fairall, E. F. Bradley, J. E. Hare, A. A. Grachev, and J. B. Edson, "Bulk Parameterization of Air Sea Fluxes: Updates and Verification for the COARE Algorithm," J. Clim., vol. 16, pp. 571-591, 2003.

[27] F. T. Ulaby, R. K. Moore, and A. K. Fung, Microwave Remote Sensing: Active and Passive. Dedham, USA: Artech House, 1986, vol. 3, pp. 1065-2126.

[28] S. A. Grodsky, V. N. Kudryavtsev, A. Bentamy, J. A. Carton, and B. Chapron, "Does direct impact of SST on short wind waves matter for scatterometry?" Geophys. Res. Lett., vol. 39, no. 12, 2012, doi: 10.1029/2012GL052091.

[29] J. C. Lagarias, J. A. Reeds, M. H. Wright, and P. E. Wright, "Convergence Properties of the Nelder-Mead Simplex Method in Low Dimensions," SIAM J. Optim., vol. 9, no. 1, pp. 112-147, 1998.

[30] NSCAT-4 geophysical model function. Web page. Royal Netherlands Meteorological Institute (KNMI). [Online]. Available: http://projects.knmi.nl/scatterometer/nscat_gmf/

[31] V. N. Kudryavtsev, B. Chapron, A. G. Myasoedov, F. Collard, and J. A. Johannessen, "On Dual Co-Polarized SAR Measurements of the Ocean Surface," IEEE Geosci. Remote Sens. Lett., vol. 10, pp. 761-765, 2013.

[32] W. J. Plant, "A two-scale model of short wind-generated waves and scatterometry," J. Geophys. Res. Oceans, vol. 91, pp. 10735-10749, 1986.

[33] V. Kudryavtsev, D. Hauser, G. Caudal, and B. Chapron, "A semiempirical model of the normalized radar cross-section of the sea surface 1. Background model," J. Geophys. Res. Oceans, vol. 108, no. C3, pp. FET 2-1-FET 2-24, 2003, doi: 10.1029/2001JC001003.

[34] G. R. Valenzuela, "Theories for the interaction of electromagnetic and ocean waves - a review," Bound.-Layer Meteor., vol. 13, pp. 61-85, 
1978.

[35] T. Elfouhaily, D. R. Thompson, D. Vandemark, and B. Chapron, "A new bistatic model for electromagnetic scattering from perfectly conducting random surfaces," Waves Random Media, vol. 9, no. 3, pp. 281-294, 1999.

[36] O. M. Phillips, Dynamics of the Upper Ocean. Cambridge-London-New York-Melbourne: Cambridge Univ. Press, 1977.

[37] A. A. Mouche, D. Hauser, and V. Kudryavtsev, "Radar scattering of the ocean surface and sea-roughness properties: A combined analysis from dual-polarizations airborne radar observations and models in C band," J. Geophys. Res. Oceans, vol. 111, no. C10, 2006, doi: 10.1029/2005JC003166.

[38] M. V. Yurovskaya, V. A. Dulov, B. Chapron, and V. N. Kudryavtsev, "Directional short wind wave spectra derived from the sea surface photography," J. Geophys. Res. Oceans, vol. 118, pp. 4380-4394, 2013.

[39] N. J. M. Laxague, B. K. Haus, D. Bogucki, and T. Ozgokmen, "Spectral characterization of fine-scale wind waves using shipboard optical polarimetry," J. Geophys. Res. Oceans, vol. 120, no. 4, pp. 3140-3156, 2015.

[40] E. C. Monahan and D. K. Woolf, "Comments on "Variations of Whitecap Coverage with Wind stress and Water Temperature"," J. Phys. Oceanogr., vol. 19, pp. 706-709, 1989.

[41] V. Kudryavtsev, I. Kozlov, B. Chapron, and J. A. Johannessen, "Quad-polarization SAR features of ocean currents," J. Geophys. Res. Oceans, vol. 119, no. 9, pp. 6046-6065, 2014, doi: 10.1002/2014JC010173.

[42] B. Jähne and K. S. Riemer, “Two-dimensional wave number spectra of small-scale water surface waves," J. Geophys. Res. Oceans, vol. 95, no. C7, pp. $11531-11546,1990$.

[43] S. R. Cloude and E. Pottier, "A review of target decomposition theorems in radar polarimetry," IEEE Trans. Geosci. Remote Sens., vol. 34, no. 2, pp. 498-518, 1996.

[44] J. C. Souyris and S. Mingot, "Polarimetry based on one transmitting and two receiving polarizations: the $\pi / 4$ mode," in Proc. Int. Geosci. Rem. Sens. Symp. IGARSS'02, vol. 1, Jun. 2002, pp. 629-631.

[45] J. W. Sapp, S. O. Alsweiss, Z. Jelenak, P. S. Chang, S. J. Frasier, and J. Carswell, "Airborne Co-polarization and Cross-Polarization Observations of the Ocean-Surface NRCS at C-Band," IEEE Trans. Geosci. Remote Sens., vol. 54, no. 10, pp. 5975-5992, 2016.

[46] S. H. Yueh, W. J. Wilson, and S. Dinardo, "Polarimetric radar remote sensing of ocean surface wind," IEEE Trans. Geosci. Remote Sens., vol. 40, no. 4, pp. 793-800, 2002.

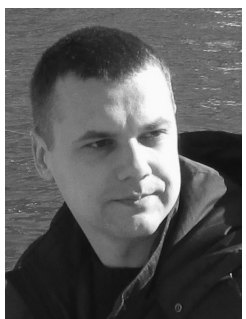

Yury Yurovsky was born in Yalta, Crimea, USSR in 1984. He received the M.S. degree in Geophysics from the Black Sea Branch of Moscow State University, Sevastopol, Ukraine in 2006. Since 2007 he works at Marine Hydrophysical Institute (MHI), Sevastopol, Russia, from where he received the Ph.D. degree in Marine physics in 2012. Currently he holds Researcher position at MHI with participating in activities at Black Sea Research platform. His work mainly includes obtaining and analysis of the data in various field experiments. His scientific interests include ocean radar and optical remote sensing, sea surface dynamics, air-sea interaction. 

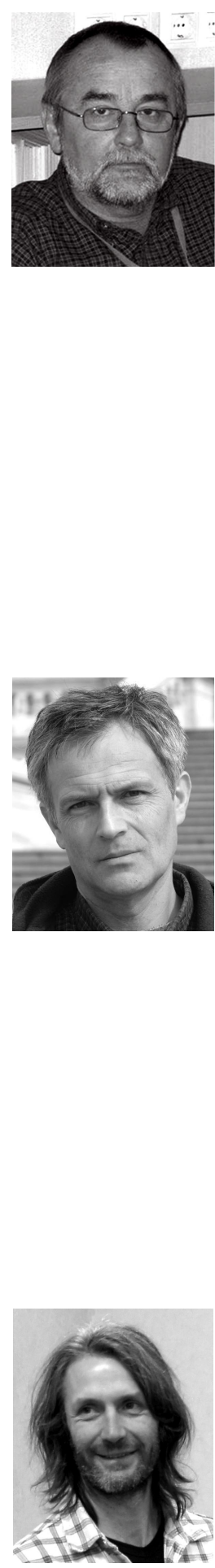

Vladimir Kudryavtsev received the Ph.D. degree and the Senior Doctorate degree in geophysics/marine physics from the Marine Hydrophysical Institute (MHI), Sevastopol, Crimea, in 1981 and 1991, respectively. In 1976, he joined MHI, where he is currently a Part-Time Principal Scientist. Since 2002, he has been a Professor at the Russian State Hydrometeorological University, St. Petersburg, where he is also the Head of the Satellite Oceanography Laboratory. From 2005 to 2013, he was with the Nansen Centre, St.Petersburg, Russia. He also currently holds a part-time senior position II with the Nansen Centre, Bergen, Norway. His current research interests include air-sea interaction, atmospheric and oceanic boundary layers, and radar and optical imaging of the ocean surface.

Semyon Grodsky received the Ph.D. degree in geophysics from the Marine Hydrophysical Institute (MHI), Sevastopol, Crimea, in 1986. He worked at MHI through 1999 before joining the Atmospheric and Oceanic Science Department, University of Maryland/College Park where he is currently a senior research scientist. His research interests include satellite remote sensing, air-sea interactions, and various aspects of ocean data analysis.

Bertrand Chapron was born in Paris, France, in 1962. He received the B.Eng. degree from the Institut National Polytechnique de Grenoble, Grenoble, France, in 1984 and the Doctorat National (Ph.D.) degree in fluid mechanics from the University of Aix-Marseille II, Marseille, France, in 1988. He spent three years as a Post-Doctoral Research Associate at the NASA/GSFC/Wallops Flight Facility, Wallops Island, VA. He has experience in applied mathematics, physical oceanography, electromagnetic waves theory, and its application to ocean remote sensing. He is currently responsible for the Oceanography from Space Laboratory, IFREMER, Plouzané, France. 